Supplement of Geosci. Model Dev., 15, 595-616, 2022

https://doi.org/10.5194/gmd-15-595-2022-supplement

(C) Author(s) 2022. CC BY 4.0 License.

(c) (i)

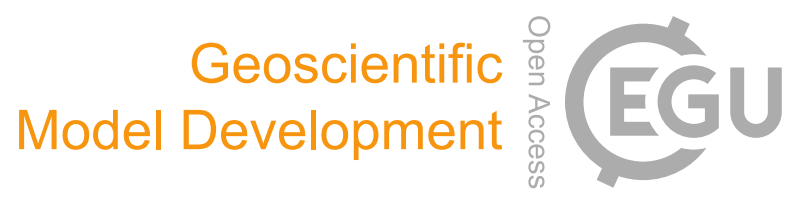

Supplement of

\title{
Afforestation impact on soil temperature in regional climate model simulations over Europe
}

\author{
Giannis Sofiadis et al. \\ Correspondence to: Giannis Sofiadis (sofiadis@geo.auth.gr)
}

The copyright of individual parts of the supplement might differ from the article licence. 


\section{Supplementary material}

Table S1: Names and characteristics of the RCMs used from Davin et al. 2020.

\begin{tabular}{|c|c|c|c|c|c|c|c|c|c|}
\hline $\begin{array}{l}\text { Model } \\
\text { name }\end{array}$ & $\begin{array}{l}\text { CCLM- } \\
\text { TERRA }\end{array}$ & $\begin{array}{l}\text { CCLM- } \\
\text { VEG3D }\end{array}$ & $\begin{array}{l}\text { CCLM- } \\
\text { CLM4.5 }\end{array}$ & RCA & $\begin{array}{l}\text { RegCM- } \\
\text { CLM4.5 }\end{array}$ & $\begin{array}{l}\text { REMO- } \\
\text { iMOVE }\end{array}$ & $\begin{array}{l}\text { WRFa- } \\
\text { NoahMP }\end{array}$ & $\begin{array}{l}\text { WRFb- } \\
\text { NoahMP }\end{array}$ & $\begin{array}{l}\text { WRFb- } \\
\text { CLM4.0 }\end{array}$ \\
\hline $\begin{array}{l}\text { Institute } \\
\text { ID }\end{array}$ & $\begin{array}{l}\text { JLU/BTU/ } \\
\text { CMCC }\end{array}$ & KIT & ETH & SMHI & ICTP & GERICS & IDL & $\mathrm{UHOH}$ & AUTH \\
\hline RCM & $\begin{array}{l}\text { COSMO_ } \\
5.0 \_c l m 9\end{array}$ & $\begin{array}{l}\text { COSMO_- } \\
5.0 \_c l m 9\end{array}$ & $\begin{array}{l}\text { COSMO } \\
5.0 \_\mathrm{clm} 9\end{array}$ & RCA4 & $\begin{array}{l}\text { RegCM4.6 } \\
.1 \text { (Giorgi } \\
\text { et al. } \\
\text { 2012) }\end{array}$ & $\begin{array}{l}\text { REMO200 } \\
9\end{array}$ & WRF381 & WRF381 & WRF381 \\
\hline $\begin{array}{l}\text { Land } \\
\text { surface } \\
\text { scheme }\end{array}$ & $\begin{array}{l}\text { TERRA- } \\
\text { ML } \\
\text { (Schrodin } \\
\text { and Heise } \\
\text { 2002) }\end{array}$ & $\begin{array}{l}\text { VEG3D } \\
\text { (Breil et } \\
\text { al. 2018) }\end{array}$ & $\begin{array}{l}\text { CLM4.5 } \\
\text { (Oleson et } \\
\text { al. 2013) }\end{array}$ & $\begin{array}{l}\text { (Samuelss } \\
\text { on et al. } \\
2006)\end{array}$ & $\begin{array}{l}\text { CLM4.5 } \\
\text { (Oleson et } \\
\text { al. 2013) }\end{array}$ & $\begin{array}{l}\text { iMOVE } \\
\text { (Wilhelm } \\
\text { et al. } \\
\text { 2014) }\end{array}$ & NoahMP & NoahMP & $\begin{array}{l}\text { CLM4.0 } \\
\text { (Oleson et } \\
\text { al. 2008) }\end{array}$ \\
\hline $\begin{array}{l}\text { Land } \\
\text { cover } \\
\text { classes } \\
\text { (classes } \\
\text { effectively } \\
\text { used in } \\
\text { FOREST } \\
\text { and } \\
\text { GRASS in } \\
\text { bold) }\end{array}$ & $\begin{array}{l}\text { 1: everg. } \\
\text { br. forest } \\
\text { 2: desc. } \\
\text { broad } \\
\text { closed } \\
\text { 3: desc. } \\
\text { br. open } \\
\text { 4: everg. } \\
\text { Needleleaf } \\
\text { forest } \\
\text { 5: desc. } \\
\text { needleleaf } \\
\text { forest } \\
\text { 6: mixed } \\
\text { leaf trees } \\
\text { 7: fresh } \\
\text { water } \\
\text { flooded } \\
\text { trees } \\
\text { 8: saline } \\
\text { water } \\
\text { flooded } \\
\text { trees } \\
\text { 9: mosaic } \\
\text { tree/natura } \\
\text { 1 veget. } \\
\text { 10: burnt } \\
\text { tree cover } \\
\text { 11: everg. } \\
\text { shupbs } \\
\text { closed/ope } \\
\text { n } \\
\text { 12: desc. } \\
\text { shrubs } \\
\text { closed/ope } \\
\text { n } \\
\text { 13: herbac. } \\
\text { veget. } \\
\text { closed/ope } \\
\text { n } \\
\text { 14: grass } \\
\text { 15: } \\
\text { flooded } \\
\text { shrups or } \\
\text { herbac. } \\
\text { 16: } \\
\text { cultivated } \\
\text { and }\end{array}$ & $\begin{array}{l}\text { 1: bare } \\
\text { soil } \\
2: \text { water } \\
3: \text { urban } \\
\text { 4: } \\
\text { deciduous } \\
\text { forest } \\
\text { 5: } \\
\text { coniferous } \\
\text { forest } \\
6: \text { mixed } \\
\text { forest } \\
7: \\
\text { cropland } \\
\text { 8: special } \\
\text { crops } \\
\text { 9: } \\
\text { grassland } \\
\text { 10: } \\
\text { shrubland }\end{array}$ & $\begin{array}{l}\text { 1: Bare } \\
\text { Soil } \\
\text { 2: } \\
\text { Needleleaf } \\
\text { Evergreen } \\
\text { Tree - } \\
\text { Temperat } \\
\text { e } \\
\text { 3: } \\
\text { Needleleaf } \\
\text { Evergreen } \\
\text { Tree - } \\
\text { Boreal } \\
\text { 4: } \\
\text { Needleleaf } \\
\text { Deciduous } \\
\text { Tree - } \\
\text { Boreal } \\
\text { 5: } \\
\text { Broadleaf } \\
\text { Evergreen } \\
\text { Tree - } \\
\text { Tropical } \\
\text { 6: } \\
\text { Broadleaf } \\
\text { Evergreen } \\
\text { Tree - } \\
\text { Temperat } \\
\text { e } \\
\text { 7: } \\
\text { Broadleaf } \\
\text { Deciduous } \\
\text { Tree - } \\
\text { Tropical } \\
\text { 8: } \\
\text { Broadleaf } \\
\text { Deciduous } \\
\text { Tree - } \\
\text { Temperat } \\
\text { e } \\
\text { 9: } \\
\text { Broadleaf } \\
\text { Deciduous } \\
\text { Tree - } \\
\text { Boreal } \\
\text { 10: } \\
\text { Broadleaf } \\
\text { Deciduous }\end{array}$ & $\begin{array}{l}\text { 1: bare } \\
\text { soil } \\
2: \text { open } \\
\text { land } \\
\text { 3: needle } \\
\text { leaf forest } \\
\text { 4: broad } \\
\text { leaf forest }\end{array}$ & $\begin{array}{l}\text { 1: Bare } \\
\text { Soil } \\
\text { 2: } \\
\text { Needleleaf } \\
\text { Evergreen } \\
\text { Tree - } \\
\text { Temperat } \\
\text { e } \\
\text { 3: } \\
\text { Needleleaf } \\
\text { Evergreen } \\
\text { Tree - } \\
\text { Boreal } \\
\text { 4: } \\
\text { Needleleaf } \\
\text { Deciduous } \\
\text { Tree - } \\
\text { Boreal } \\
\text { 5: } \\
\text { Broadleaf } \\
\text { Evergreen } \\
\text { Tree - } \\
\text { Tropical } \\
\text { 6: } \\
\text { Broadleaf } \\
\text { Evergreen } \\
\text { Tree - } \\
\text { Temperat } \\
\text { e } \\
\text { 7: } \\
\text { Broadleaf } \\
\text { Deciduous } \\
\text { Tree - } \\
\text { Tropical } \\
\text { 8: } \\
\text { Broadleaf } \\
\text { Deciduous } \\
\text { Tree - } \\
\text { Temperat } \\
\text { e } \\
\text { 9: } \\
\text { Broadleaf } \\
\text { Deciduous } \\
\text { Tree - } \\
\text { Boreal } \\
\text { 10: } \\
\text { Broadleaf } \\
\text { Deciduous }\end{array}$ & $\begin{array}{l}\text { 1: tr. br. } \\
\text { everg. } \\
\text { 2: tr. br. } \\
\text { desc. } \\
\text { 3: temp. } \\
\text { br. everg. } \\
\text { 4: temp. } \\
\text { br. desc. } \\
\text { 5: everg. } \\
\text { conif. } \\
\text { 6: desc. } \\
\text { conif. } \\
\text { 7: everg. } \\
\text { shrubs } \\
\text { 8: desc. } \\
\text { shrubs } \\
\text { 9: C3 } \\
\text { grasses } \\
\text { 10: C4 } \\
\text { grasses } \\
\text { 11: tundra } \\
\text { 12: } \\
\text { swamps } \\
\text { 13: C3 } \\
\text { crops } \\
\text { 14: C4 } \\
\text { crops } \\
\text { 15: urban } \\
\text { 16: bare }\end{array}$ & $\begin{array}{l}\text { 1: everg. } \\
\text { needleleaf } \\
\text { 2: desc. } \\
\text { Needleleaf } \\
\text { 3: everg. } \\
\text { Broadleaf } \\
\text { 4: desc. } \\
\text { Broadleaf } \\
\text { 5: mixed } \\
\text { forests } \\
\text { 6: closed } \\
\text { shrubland } \\
\text { 7: open } \\
\text { shrubland } \\
\text { 8: wooded } \\
\text { savannah } \\
\text { 9: } \\
\text { savannah } \\
\text { 10: } \\
\text { grassland } \\
\text { 11: } \\
\text { wetlands } \\
\text { 12: } \\
\text { cropland } \\
\text { 13: urban } \\
\text { and built- } \\
\text { up } \\
\text { 14: } \\
\text { cropland/n } \\
\text { atural } \\
\text { vegetation } \\
\text { mosaic } \\
\text { 15: snow } \\
\text { and ice } \\
\text { 16: } \\
\text { barren or } \\
\text { sparsely } \\
\text { vegetated } \\
\text { 17: water } \\
\text { 18: } \\
\text { wooded } \\
\text { tundra } \\
\text { 19: mixed } \\
\text { tundra } \\
\text { 20: barren } \\
\text { tundra } \\
\text { 21: lakes }\end{array}$ & $\begin{array}{l}\text { 1: everg. } \\
\text { needleleaf } \\
\text { 2: desc. } \\
\text { Needleleaf } \\
\text { 3: everg. } \\
\text { Broadleaf } \\
\text { 4: desc. } \\
\text { Broadleaf } \\
\text { 5: mixed } \\
\text { forests } \\
\text { 6: closed } \\
\text { shrubland } \\
\text { 7: open } \\
\text { shrubland } \\
8: \text { wooded } \\
\text { savannah } \\
\text { 9: } \\
\text { savannah } \\
\text { 10: } \\
\text { grassland } \\
\text { 11: } \\
\text { wetlands } \\
\text { 12: } \\
\text { cropland } \\
\text { 13: urban } \\
\text { and built- } \\
\text { up } \\
\text { 14: } \\
\text { cropland/n } \\
\text { atural } \\
\text { vegetation } \\
\text { mosaic } \\
\text { 15: snow } \\
\text { and ice } \\
\text { 16: } \\
\text { barren or } \\
\text { sparsely } \\
\text { vegetated } \\
\text { 17: water } \\
\text { 18: } \\
\text { wooded } \\
\text { tundra } \\
\text { 19: mixed } \\
\text { tundra } \\
\text { 20: barren } \\
\text { tundra } \\
\text { 21: lakes }\end{array}$ & $\begin{array}{l}\text { 1: Bare } \\
\text { Soil } \\
\text { 2: } \\
\text { Needleleaf } \\
\text { Evergreen } \\
\text { Tree - } \\
\text { Temperat } \\
\text { e } \\
\text { 3: } \\
\text { Needleleaf } \\
\text { Evergreen } \\
\text { Tree - } \\
\text { Boreal } \\
\text { 4: } \\
\text { Needleleaf } \\
\text { Deciduous } \\
\text { Tree - } \\
\text { Boreal } \\
\text { 5: } \\
\text { Broadleaf } \\
\text { Evergreen } \\
\text { Tree - } \\
\text { Tropical } \\
\text { 6: } \\
\text { Broadleaf } \\
\text { Evergreen } \\
\text { Tree - } \\
\text { Temperat } \\
\text { e } \\
\text { 7: } \\
\text { Broadleaf } \\
\text { Deciduous } \\
\text { Tree - } \\
\text { Tropical } \\
\text { 8: } \\
\text { Broadleaf } \\
\text { Deciduous } \\
\text { Tree - } \\
\text { Temperat } \\
\text { e } \\
\text { 9: } \\
\text { Broadleaf } \\
\text { Deciduous } \\
\text { Tree - } \\
\text { Boreal } \\
\text { 10: } \\
\text { Broadleaf } \\
\text { Deciduous }\end{array}$ \\
\hline
\end{tabular}




\begin{tabular}{|c|c|c|c|c|c|c|c|c|c|}
\hline & $\begin{array}{l}\text { managed } \\
\text { 17: mosaic } \\
\text { crop/tree/n } \\
\text { et veget. } \\
\text { 18: mosaic } \\
\text { crop/shrub } \\
\text { /grass } \\
\text { 19: bare } \\
\text { areas } \\
\text { 20: water } \\
21: \text { snow } \\
\text { and ice } \\
22 . \\
\text { artificial } \\
\text { surface } \\
23: \\
\text { undefined }\end{array}$ & & $\begin{array}{l}\text { Shrub - } \\
\text { Temperate } \\
\text { 11: } \\
\text { Broadleaf } \\
\text { Evergreen } \\
\text { Shrub - } \\
\text { Temperate } \\
\text { 12: } \\
\text { Broadleaf } \\
\text { Deciduous } \\
\text { Shrub - } \\
\text { Boreal } \\
\text { 13: C3 } \\
\text { artic grass } \\
\text { 14: C3 } \\
\text { grass } \\
\text { 15: C4 } \\
\text { grass } \\
\text { 16: Crop } 1 \\
\text { 17: Crop } 2\end{array}$ & & $\begin{array}{l}\text { Shrub - } \\
\text { Temperate } \\
\text { 11: } \\
\text { Broadleaf } \\
\text { Evergreen } \\
\text { Shrub - } \\
\text { Temperate } \\
\text { 12: } \\
\text { Broadleaf } \\
\text { Deciduous } \\
\text { Shrub - } \\
\text { Boreal } \\
\text { 13: C3 } \\
\text { artic grass } \\
\text { 14: C3 } \\
\text { grass } \\
\text { 15: C4 } \\
\text { grass } \\
\text { 16: Crop } 1 \\
\text { 17: Crop } 2\end{array}$ & & & & $\begin{array}{l}\text { Shrub - } \\
\text { Temperate } \\
\text { 11: } \\
\text { Broadleaf } \\
\text { Evergreen } \\
\text { Shrub - } \\
\text { Temperate } \\
\text { 12: } \\
\text { Broadleaf } \\
\text { Deciduous } \\
\text { Shrub - } \\
\text { Boreal } \\
\text { 13: C3 } \\
\text { artic grass } \\
\text { 14: C3 } \\
\text { grass } \\
\text { 15: C4 } \\
\text { grass } \\
\text { 16: Crop1 } \\
\text { 17: Crop2 }\end{array}$ \\
\hline $\begin{array}{l}\text { Conversio } \\
\text { n method } \\
\text { to } \\
\text { implemen } \\
\text { t the PFT- } \\
\text { based } \\
\text { input } \\
\text { vegetation } \\
\text { maps } \\
\text { (FOREST } \\
\text { and } \\
\text { GRASS) }\end{array}$ & $\begin{array}{l}\text { bare } \\
\text { soil=19 } \\
\text { Needleleaf } \\
\text { evergreen } \\
\text { tree- } \\
\text { temperate= } \\
4 \\
\text { Needleleaf } \\
\text { evergreen } \\
\text { tree- } \\
\text { boreal=4 } \\
\text { Needleleaf } \\
\text { deciduous } \\
\text { tree- } \\
\text { boreal=5 } \\
\text { Broadleaf } \\
\text { evergreen } \\
\text { tree - } \\
\text { tropical=1 } \\
\text { Broadleaf } \\
\text { evergreen } \\
\text { tree }- \\
\text { temperate= } \\
1 \\
\text { Broadleaf } \\
\text { deciduous } \\
\text { tree }- \\
\text { tropical=2 } \\
\text { Broadleaf } \\
\text { deciduous } \\
\text { tree }- \\
\text { temperate= } \\
2 \\
\text { Broadleaf } \\
\text { deciduous } \\
\text { tree }- \\
\text { boreal=3 } \\
\text { C3 arctic } \\
\text { grass= } 14 \\
\text { C3 grass= } \\
14 \\
\text { C4 grass= } \\
14\end{array}$ & $\begin{array}{l}\text { bare soil=1 } \\
\text { Needleleaf } \\
\text { evergreen } \\
\text { tree- } \\
\text { temperate= } \\
5 \\
\text { Needleleaf } \\
\text { evergreen } \\
\text { tree- } \\
\text { boreal=5 } \\
\text { Needleleaf } \\
\text { deciduous } \\
\text { tree- } \\
\text { boreal=5 } \\
\text { Broadleaf } \\
\text { evergreen } \\
\text { tree - } \\
\text { tropical=4 } \\
\text { Broadleaf } \\
\text { evergreen } \\
\text { tree }- \\
\text { temperate= } \\
4 \\
\text { Broadleaf } \\
\text { deciduous } \\
\text { tree }- \\
\text { tropical=4 } \\
\text { Broadleaf } \\
\text { deciduous } \\
\text { tree }- \\
\text { temperate= } \\
4 \\
\text { Broadleaf } \\
\text { deciduous } \\
\text { tree }- \\
\text { boreal=4 } \\
\text { C3 arctic } \\
\text { grass =9 } \\
\text { C3 grass } \\
=9 \\
\text { C4 grass } \\
=9\end{array}$ & $\begin{array}{l}\text { No } \\
\text { conversion } \\
\text { needed }\end{array}$ & $\begin{array}{l}\text { Bare soil = } \\
1 \\
\text { Needleleaf } \\
\text { evergreen } \\
\text { tree- } \\
\text { temperate } \\
=3 \\
\text { Needleleaf } \\
\text { evergreen } \\
\text { tree-boreal } \\
=3 \\
\text { Needleleaf } \\
\text { deciduous } \\
\text { tree-boreal } \\
=3 \\
\text { Broadleaf } \\
\text { evergreen } \\
\text { tree - } \\
\text { tropical = } \\
4 \\
\text { Broadleaf } \\
\text { evergreen } \\
\text { tree }- \\
\text { temperate } \\
=4 \\
\text { Broadleaf } \\
\text { deciduous } \\
\text { tree }- \\
\text { tropical = } \\
4 \\
\text { Broadleaf } \\
\text { deciduous } \\
\text { tree }- \\
\text { temperate } \\
=4 \\
\text { Broadleaf } \\
\text { deciduous } \\
\text { tree }- \\
\text { boreal = } 4 \\
\text { C3 arctic } \\
\text { grass = } 2 \\
\text { C3 grass = } \\
2 \\
\text { C4 grass = } \\
2\end{array}$ & $\begin{array}{l}\text { No } \\
\text { conversion } \\
\text { needed }\end{array}$ & 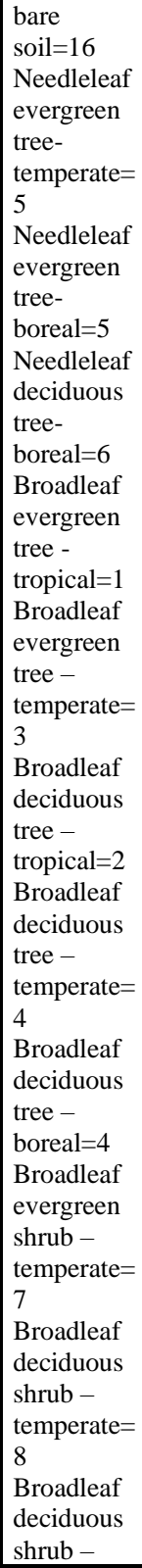 & $\begin{array}{l}\text { Bare soil = } \\
16 \\
\text { Needleleaf } \\
\text { evergreen } \\
\text { tree- } \\
\text { temperate } \\
=1 \\
\text { Needleleaf } \\
\text { evergreen } \\
\text { tree-boreal } \\
=1 \\
\text { Needleleaf } \\
\text { deciduous } \\
\text { tree-boreal } \\
=2 \\
\text { Broadleaf } \\
\text { evergreen } \\
\text { tree }- \\
\text { tropical = } \\
3 \\
\text { Broadleaf } \\
\text { evergreen } \\
\text { tree }- \\
\text { temperate } \\
=3 \\
\text { Broadleaf } \\
\text { deciduous } \\
\text { tree }- \\
\text { tropical }= \\
4 \\
\text { Broadleaf } \\
\text { deciduous } \\
\text { tree }- \\
\text { temperate } \\
=4 \\
\text { Broadleaf } \\
\text { deciduous } \\
\text { tree }- \\
\text { boreal = } 4 \\
\text { C3 arctic } \\
\text { grass }=10 \\
\text { C3 grass = } \\
10 \\
\text { C4 grass = } \\
10\end{array}$ & $\begin{array}{l}\text { Bare soil = } \\
16 \\
\text { Needleleaf } \\
\text { evergreen } \\
\text { tree- } \\
\text { temperate } \\
=1 \\
\text { Needleleaf } \\
\text { evergreen } \\
\text { tree-boreal } \\
=1 \\
\text { Needleleaf } \\
\text { deciduous } \\
\text { tree-boreal } \\
=2 \\
\text { Broadleaf } \\
\text { evergreen } \\
\text { tree - } \\
\text { tropical }= \\
3 \\
\text { Broadleaf } \\
\text { evergreen } \\
\text { tree }- \\
\text { temperate } \\
=3 \\
\text { Broadleaf } \\
\text { deciduous } \\
\text { tree }- \\
\text { tropical }= \\
4 \\
\text { Broadleaf } \\
\text { deciduous } \\
\text { tree }- \\
\text { temperate } \\
=4 \\
\text { Broadleaf } \\
\text { deciduous } \\
\text { tree }- \\
\text { boreal }=4 \\
\text { C3 arctic } \\
\text { grass = } 10 \\
\text { C3 grass = } \\
10 \\
\text { C4 grass = } \\
10 \\
\end{array}$ & $\begin{array}{l}\text { No } \\
\text { conversion } \\
\text { needed }\end{array}$ \\
\hline
\end{tabular}




\begin{tabular}{|c|c|c|c|c|c|c|c|c|c|}
\hline & & & & & & $\begin{array}{l}\text { boreal }=8 \\
\text { C3 arctic } \\
\text { grass }=9 \\
\text { C3 } \\
\text { grass }=9 \\
\text { C4 } \\
\text { grass }=10\end{array}$ & & & \\
\hline $\begin{array}{l}\text { Represent } \\
\text { ation of } \\
\text { sub-grid } \\
\text { scale } \\
\text { vegetation } \\
\text { heterogen } \\
\text { eity }\end{array}$ & $\begin{array}{l}\text { Single } \\
\text { class }\end{array}$ & $\begin{array}{l}\text { Single } \\
\text { class }\end{array}$ & $\begin{array}{l}\text { Tile } \\
\text { approach }\end{array}$ & $\begin{array}{l}\text { Tile } \\
\text { approach }\end{array}$ & $\begin{array}{l}\text { Tile } \\
\text { approach }\end{array}$ & $\begin{array}{l}\text { Tile } \\
\text { approach }\end{array}$ & $\begin{array}{l}\text { Single } \\
\text { class }\end{array}$ & $\begin{array}{l}\text { Single } \\
\text { class }\end{array}$ & $\begin{array}{l}\text { Tile } \\
\text { approach }\end{array}$ \\
\hline $\begin{array}{l}\text { Leaf Area } \\
\text { Index }\end{array}$ & $\begin{array}{l}\text { Prescribed } \\
\text { seasonal } \\
\text { cycle } \\
\text { (sinus } \\
\text { function } \\
\text { depending } \\
\text { on altitude } \\
\text { and } \\
\text { latitude } \\
\text { with } \\
\text { vegetation- } \\
\text { dependent } \\
\text { minimum } \\
\text { and } \\
\text { maximum } \\
\text { values) } \\
\end{array}$ & \begin{tabular}{|l} 
Prescribed \\
seasonal \\
cycle \\
(sinus \\
function \\
depending \\
on altitude \\
and \\
latitude \\
with \\
vegetation- \\
dependent \\
minimum \\
and \\
maximum \\
values) \\
\end{tabular} & $\begin{array}{l}\text { Prescribed } \\
\text { seasonal } \\
\text { cycle } \\
\text { based on } \\
\text { MODIS } \\
\text { (Lawrence } \\
\text { and Chase } \\
2007 \text { ) }\end{array}$ & $\begin{array}{l}\text { Calculated } \\
\text { monthly } \\
\text { based on } \\
\text { vegetation } \\
\text { type, soil } \\
\text { temperatur } \\
\text { e and soil } \\
\text { moisture }\end{array}$ & $\begin{array}{l}\text { Prescribed } \\
\text { seasonal } \\
\text { cycle } \\
\text { based on } \\
\text { MODIS } \\
\text { (Lawrence } \\
\text { and Chase } \\
\text { 2007) }\end{array}$ & $\begin{array}{l}\text { Calculated } \\
\text { daily } \\
\text { based on } \\
\text { atmospheri } \\
\text { c forcing } \\
\text { and soil } \\
\text { moisture } \\
\text { state }\end{array}$ & $\begin{array}{l}\text { Prescribed } \\
\text { seasonal } \\
\text { cycle } \\
\text { based on } \\
\text { lookup } \\
\text { tables }\end{array}$ & $\begin{array}{l}\text { Prescribed } \\
\text { seasonal } \\
\text { cycle } \\
\text { based on } \\
\text { lookup } \\
\text { tables }\end{array}$ & $\begin{array}{l}\text { Prescribed } \\
\text { seasonal } \\
\text { cycle } \\
\text { based on } \\
\text { MODIS } \\
\text { (Lawrence } \\
\text { and Chase } \\
\text { 2007) }\end{array}$ \\
\hline $\begin{array}{l}\text { Total soil } \\
\text { depth and } \\
\text { number of } \\
\text { hydrologi } \\
\text { cally/ther } \\
\text { mally } \\
\text { active soil } \\
\text { layers }\end{array}$ & $\begin{array}{l}9 \\
\text { thermally } \\
\text { active } \\
\text { layers } \\
\text { down to } \\
7.5 \mathrm{~m} \text {; first } \\
8 \\
\text { hydrologic } \\
\text { ally active } \\
\text { down to } \\
3.9 \mathrm{~m}\end{array}$ & $\begin{array}{l}9 \text { layers } \\
\text { down to } \\
7.5 \mathrm{~m}\end{array}$ & $\begin{array}{l}15 \text { layers } \\
\text { for thermal } \\
\text { calculation } \\
\mathrm{s} \text { down to } \\
42 \mathrm{~m} \text {; first } \\
10 \\
\text { hydrologic } \\
\text { ally active } \\
\text { down to } \\
3.43 \mathrm{~m}\end{array}$ & $\begin{array}{l}5 \text { layers } \\
\text { down to } \\
2.89 \mathrm{~m}\end{array}$ & $\begin{array}{l}15 \text { layers } \\
\text { for thermal } \\
\text { calculation } \\
\text { s down to } \\
42 \mathrm{~m} \text {; first } \\
10 \\
\text { hydrologic } \\
\text { ally active } \\
\text { down to } \\
3.43 \mathrm{~m}\end{array}$ & $\begin{array}{l}5 \\
\text { thermally } \\
\text { active } \\
\text { layers } \\
\text { down to } 10 \\
\text { m; } 1 \text { water } \\
\text { bucket }\end{array}$ & $\begin{array}{l}4 \text { layers } \\
\text { down to } 1 \\
\mathrm{~m}\end{array}$ & $\begin{array}{l}4 \text { layers } \\
\text { down to } 1 \\
\mathrm{~m}\end{array}$ & $\begin{array}{l}10 \text { layers } \\
\text { down to } \\
3.43 \mathrm{~m}\end{array}$ \\
\hline $\begin{array}{l}\text { Initialisati } \\
\text { on and } \\
\text { spin up }\end{array}$ & $\begin{array}{l}\text { Initializati } \\
\text { on with } \\
\text { ERA- } \\
\text { Interim, } \\
\text { 1979-1985 } \\
\text { as spin-up }\end{array}$ & $\begin{array}{l}\text { Initializati } \\
\text { on with } \\
\text { ERA- } \\
\text { Interim, } \\
\text { 1979-1985 } \\
\text { as spin-up }\end{array}$ & $\begin{array}{l}\text { Initializati } \\
\text { on with } \\
\text { ERA- } \\
\text { Interim, } \\
\text { 1979-1985 } \\
\text { as spin-up }\end{array}$ & $\begin{array}{l}\text { Initializati } \\
\text { on with } \\
\text { ERA- } \\
\text { Interim, } \\
\text { 1979-1985 } \\
\text { as spin-up }\end{array}$ & $\begin{array}{l}\text { Initializati } \\
\text { on with } \\
\text { ERA- } \\
\text { Interim } \\
\text { except soil } \\
\text { moisture } \\
\text { which is } \\
\text { based on a } \\
\text { climatolog } \\
\text { ical } \\
\text { average } \\
\text { (Giorgi et } \\
\text { al. 1989); } \\
\text { 1985 as } \\
\text { spin-up }\end{array}$ & $\begin{array}{l}\text { Initializati } \\
\text { on with } \\
\text { ERA- } \\
\text { Interim, } \\
\text { 1979-1985 } \\
\text { as spin-up }\end{array}$ & $\begin{array}{l}\text { Initializati } \\
\text { on with } \\
\text { ERA- } \\
\text { Interim, } \\
\text { 1979-1985 } \\
\text { as spin-up }\end{array}$ & $\begin{array}{l}\text { Initializati } \\
\text { on with } \\
\text { ERA- } \\
\text { Interim, } \\
\text { 1983-1985 } \\
\text { as spin-up }\end{array}$ & $\begin{array}{l}\text { Initializati } \\
\text { on with } \\
\text { ERA- } \\
\text { Interim, } \\
\text { 1984-1985 } \\
\text { as spin-up }\end{array}$ \\
\hline $\begin{array}{l}\text { Lateral } \\
\text { boundary } \\
\text { formulati } \\
\text { on }\end{array}$ & $\begin{array}{l}\text { (Davies } \\
1976)\end{array}$ & $\begin{array}{l}\text { (Davies } \\
1976 \text { ) }\end{array}$ & $\begin{array}{l}\text { (Davies } \\
1976 \text { ) }\end{array}$ & $\begin{array}{l}\text { (Davies } \\
\text { 1976) with } \\
\text { a cosine- } \\
\text { based } \\
\text { relaxation } \\
\text { function } \\
\end{array}$ & $\begin{array}{l}\text { (Giorgi et } \\
\text { al. 1993) }\end{array}$ & $\begin{array}{l}\text { (Davies } \\
1976)\end{array}$ & $\begin{array}{l}\text { exponentia } \\
1 \\
\text { relaxation }\end{array}$ & $\begin{array}{l}\text { exponentia } \\
1 \\
\text { relaxation }\end{array}$ & $\begin{array}{l}\text { expotential } \\
\text { relaxation }\end{array}$ \\
\hline $\begin{array}{l}\text { Buffer } \\
\text { (No. of } \\
\text { grid cells) }\end{array}$ & 13 & 13 & 13 & 8 & 12 & 8 & 15 & 10 & 10 \\
\hline
\end{tabular}




\begin{tabular}{|c|c|c|c|c|c|c|c|c|c|}
\hline $\begin{array}{l}\text { No. of } \\
\text { vertical } \\
\text { levels } \\
\end{array}$ & 40 & 40 & 40 & 24 & 23 & 27 & 50 & 40 & 40 \\
\hline $\begin{array}{l}\text { Turbulenc } \\
\text { e and } \\
\text { planetary } \\
\text { boundary } \\
\text { layer } \\
\text { scheme }\end{array}$ & $\begin{array}{l}\text { Level } 2.5 \\
\text { closure for } \\
\text { turbulent } \\
\text { kinetic } \\
\text { energy as } \\
\text { prognostic } \\
\text { variable } \\
\text { (Mellor } \\
\text { and } \\
\text { Yamada } \\
1982 \text { ) }\end{array}$ & $\begin{array}{l}\text { Level } 2.5 \\
\text { closure for } \\
\text { turbulent } \\
\text { kinetic } \\
\text { energy as } \\
\text { prognostic } \\
\text { variable } \\
\text { (Mellor } \\
\text { and } \\
\text { Yamada } \\
1982 \text { ) }\end{array}$ & $\begin{array}{l}\text { Level } 2.5 \\
\text { closure for } \\
\text { turbulent } \\
\text { kinetic } \\
\text { energy as } \\
\text { prognostic } \\
\text { variable } \\
\text { (Mellor } \\
\text { and } \\
\text { Yamada } \\
1982 \text { ) }\end{array}$ & $\begin{array}{l}\text { (Vogeleza } \\
\text { ng and } \\
\text { Holtslag } \\
1996 \text { ) }\end{array}$ & $\begin{array}{l}\text { The } \\
\text { University } \\
\text { of } \\
\text { Washingto } \\
\mathrm{n} \\
\text { turbulence } \\
\text { closure } \\
\text { model } \\
\text { (Grenier et } \\
\text { al. 2001; } \\
\text { Bretherton } \\
\text { et al. } \\
\text { 2004) }\end{array}$ & $\begin{array}{l}\text { Vertical } \\
\text { diffusion } \\
\text { after } \\
\text { (Louis } \\
\text { 1979) for } \\
\text { the Prandtl } \\
\text { layer, } \\
\text { extended } \\
\text { level-2 } \\
\text { scheme } \\
\text { after } \\
\text { (Mellor } \\
\text { and } \\
\text { Yamada } \\
\text { 1974) in } \\
\text { the Ekman } \\
\text { layer and } \\
\text { the free } \\
\text { atmospher } \\
\text { e including } \\
\text { modificati } \\
\text { ons in the } \\
\text { presence } \\
\text { of clouds }\end{array}$ & $\begin{array}{l}\text { MYNN } \\
\text { Level } 2.5 \\
\text { PBL } \\
\text { (Nakanishi } \\
\text { and Niino } \\
\text { 2006; } \\
\text { NAKANIS } \\
\text { HI and } \\
\text { NIINO } \\
2009 \text { ) }\end{array}$ & $\begin{array}{l}\text { MYNN } \\
\text { Level } 2.5 \\
\text { PBL } \\
\text { (Nakanishi } \\
\text { and Niino } \\
\text { 2006; } \\
\text { NAKANIS } \\
\text { HI and } \\
\text { NIINO } \\
2009 \text { ) }\end{array}$ & $\begin{array}{l}\text { MYNN } \\
\text { Level } 2.5 \\
\text { PBL } \\
\text { (Nakanishi } \\
\text { and Niino } \\
\text { 2006; } \\
\text { NAKANIS } \\
\text { HI and } \\
\text { NIINO } \\
\text { 2009) }\end{array}$ \\
\hline $\begin{array}{l}\text { Radiation } \\
\text { scheme }\end{array}$ & $\begin{array}{l}\text { (Ritter et } \\
\text { al. 1992) }\end{array}$ & $\begin{array}{l}\text { (Ritter et } \\
\text { al. 1992) }\end{array}$ & $\begin{array}{l}\text { (Ritter et } \\
\text { al. 1992) }\end{array}$ & $\begin{array}{l}\text { (Savijärvi } \\
\text { and } \\
\text { Savijärvi } \\
\text { 1990), } \\
\text { Wyser et } \\
\text { al (1999) }\end{array}$ & 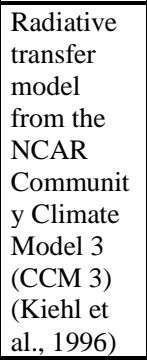 & $\begin{array}{l}\text { (Morcrette } \\
\text { et al. } \\
\text { 1986) with } \\
\text { modificati } \\
\text { ons for } \\
\text { additional } \\
\text { greenhous } \\
\text { e gases, } \\
\text { ozone and } \\
\text { various } \\
\text { aerosols. }\end{array}$ & $\begin{array}{l}\text { Rapid } \\
\text { Radiative } \\
\text { Transfer } \\
\text { Model } \\
\text { (RRTMG) } \\
\text { scheme } \\
\text { (Iacono et } \\
\text { al. 2008) }\end{array}$ & $\begin{array}{l}\text { Rapid } \\
\text { Radiative } \\
\text { Transfer } \\
\text { Model } \\
\text { (RRTMG) } \\
\text { scheme } \\
\text { (Iacono et } \\
\text { al. 2008) }\end{array}$ & $\begin{array}{l}\text { Rapid } \\
\text { Radiative } \\
\text { Transfer } \\
\text { Model } \\
\text { (RRTMG) } \\
\text { scheme } \\
\text { (Iacono et } \\
\text { al. 2008) }\end{array}$ \\
\hline $\begin{array}{l}\text { Convectio } \\
\text { n } \\
\text { scheme }\end{array}$ & $\begin{array}{l}\text { (Tiedtke } \\
\text { 1989) }\end{array}$ & $\begin{array}{l}\text { (Tiedtke } \\
\text { 1989) }\end{array}$ & $\begin{array}{l}\text { (Tiedtke } \\
\text { 1989) }\end{array}$ & $\begin{array}{l}\text { (Bechtold } \\
\text { et al. } \\
\text { 2001) }\end{array}$ & $\begin{array}{l}\text { (Tiedtke } \\
\text { 1996) for } \\
\text { cumulus } \\
\text { convection }\end{array}$ & $\begin{array}{l}\text { (Tiedtke } \\
\text { 1989) with } \\
\text { modificati } \\
\text { ons after } \\
\text { Nordeng } \\
\text { (1994) }\end{array}$ & $\begin{array}{l}\text { (Grell and } \\
\text { Freitas } \\
\text { 2014) for } \\
\text { cumulus } \\
\text { convection } \\
\text { and } \\
\text { Global/Re } \\
\text { gional } \\
\text { Integrated } \\
\text { Modeling } \\
\text { System } \\
\text { (GRIMS) } \\
\text { Scheme } \\
\text { (Hong et } \\
\text { al. 2013) } \\
\text { for } \\
\text { shallow } \\
\text { convection } \\
\end{array}$ & $\begin{array}{l}\text { (Kain and } \\
\text { Fritsch } \\
\text { 1990); no } \\
\text { shallow } \\
\text { convection }\end{array}$ & $\begin{array}{l}\text { (Kain and } \\
\text { Fritsch } \\
\text { 1990); no } \\
\text { shallow } \\
\text { convection }\end{array}$ \\
\hline $\begin{array}{l}\text { Microphy } \\
\text { sics } \\
\text { scheme }\end{array}$ & $\begin{array}{l}\text { One- } \\
\text { moment } \\
\text { cloud } \\
\text { microphysi } \\
\text { cs scheme } \\
\text { (Seifert } \\
\text { and } \\
\text { Beheng } \\
2001 \text { ) } \\
\end{array}$ & \begin{tabular}{|l|} 
One- \\
moment \\
cloud \\
microphysi \\
cs scheme \\
(Seifert \\
and \\
Beheng \\
2001) \\
\end{tabular} & $\begin{array}{l}\text { One- } \\
\text { moment } \\
\text { cloud } \\
\text { microphysi } \\
\text { cs scheme } \\
\text { (Seifert } \\
\text { and } \\
\text { Beheng } \\
\text { 2001) } \\
\end{array}$ & $\begin{array}{l}\text { Values } \\
\text { from } \\
\text { tables }\end{array}$ & $\begin{array}{l}\text { Subgrid } \\
\text { Explicit } \\
\text { Moisture } \\
\text { scheme } \\
\text { (SUBEX) } \\
\text { (Pal et al. } \\
2000 \text { ) }\end{array}$ & $\begin{array}{l}\text { (Sundqvist } \\
\text { 1978)(Roe } \\
\text { ckner et } \\
\text { al., 1996) }\end{array}$ & $\begin{array}{l}\text { Two- } \\
\text { moment, } \\
\text { 6-class } \\
\text { scheme } \\
\text { (Lim and } \\
\text { Hong } \\
2010 \text { ) }\end{array}$ & $\begin{array}{l}\text { (Thompso } \\
\text { n et al. } \\
\text { 2004) }\end{array}$ & $\begin{array}{l}\text { (Thompso } \\
\mathrm{n} \text { et al. } \\
\text { 2004) }\end{array}$ \\
\hline $\begin{array}{l}\text { Greenhou } \\
\text { se gases }\end{array}$ & Historical & Historical & Historical & Historical & Historical & Historical & Historical & Constant & Constant \\
\hline
\end{tabular}




\begin{tabular}{|l|l|l|l|l|l|l|l|l|l|}
\hline \multirow{4}{*}{ Aerosols } & $\begin{array}{l}\text { Constant } \\
\text { (Tanré, } \\
1984)\end{array}$ & $\begin{array}{l}\text { (Tegen et } \\
\text { al. 1997) } \\
\text { climatolog } \\
\mathrm{y}\end{array}$ & $\begin{array}{l}\text { Constant } \\
\text { (Tanré, } \\
1984)\end{array}$ & Constant & $\begin{array}{l}\text { Not } \\
\text { accounted } \\
\text { for }\end{array}$ & $\begin{array}{l}\text { Constant } \\
\text { (Teichman } \\
\text { n et al. } \\
\text { 2013) }\end{array}$ & $\begin{array}{l}\text { (Tegen et } \\
\text { al. 1997) } \\
\text { climatolog } \\
\text { y }\end{array}$ & $\begin{array}{l}\text { (Tegen et } \\
\text { al. 1997) } \\
\text { climatolog } \\
\text { y }\end{array}$ & $\begin{array}{l}\text { (Tegen et } \\
\text { al. 1997) } \\
\text { climatolog } \\
\text { y }\end{array}$ \\
\hline
\end{tabular}

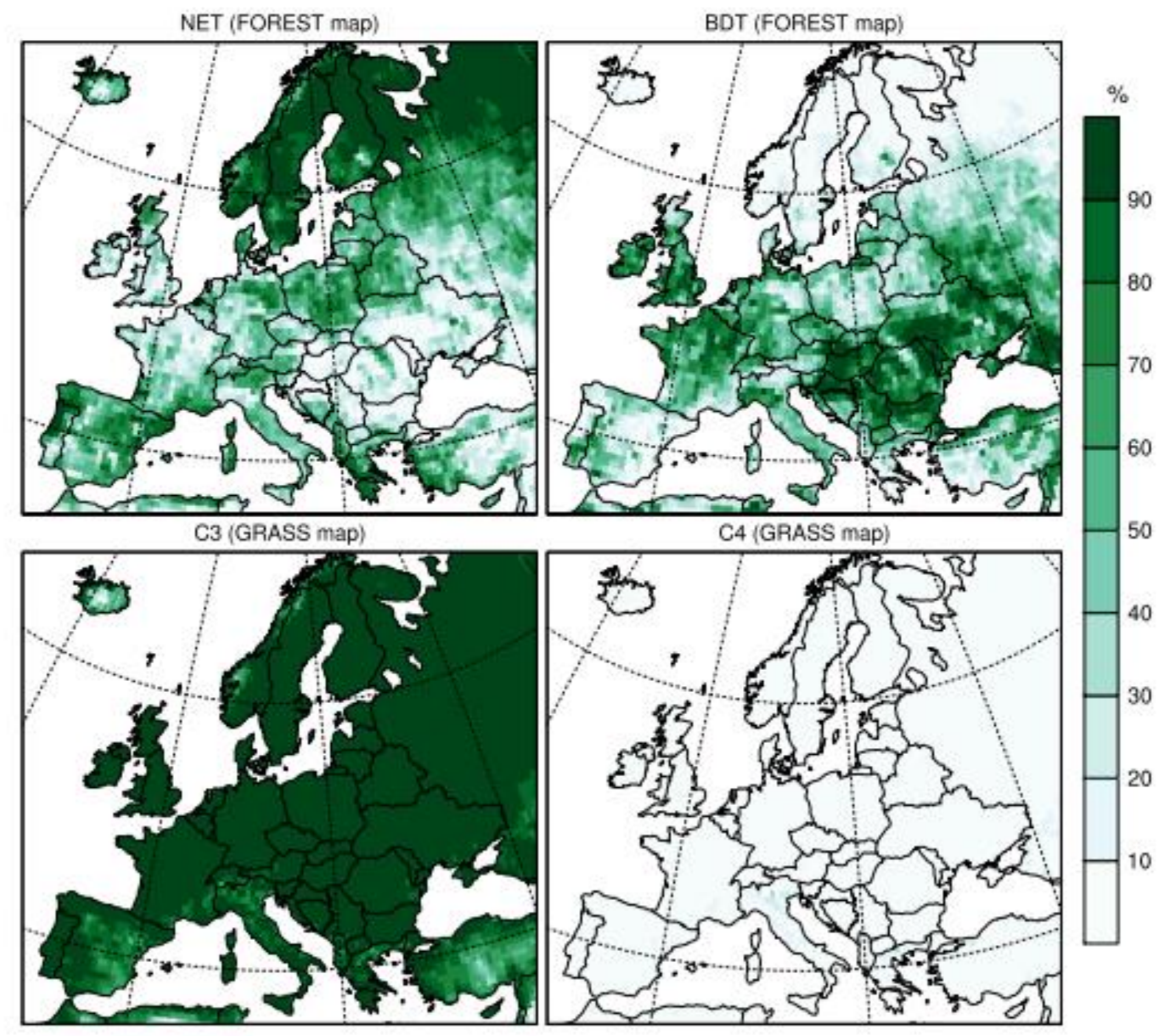

Figure S1: Vegetation maps used in the GRASS and FOREST simulations in Davin et al. 2020. NET $=$ needleleaf evergreen trees, $\mathrm{BDT}=$ broadleaf deciduous trees. 


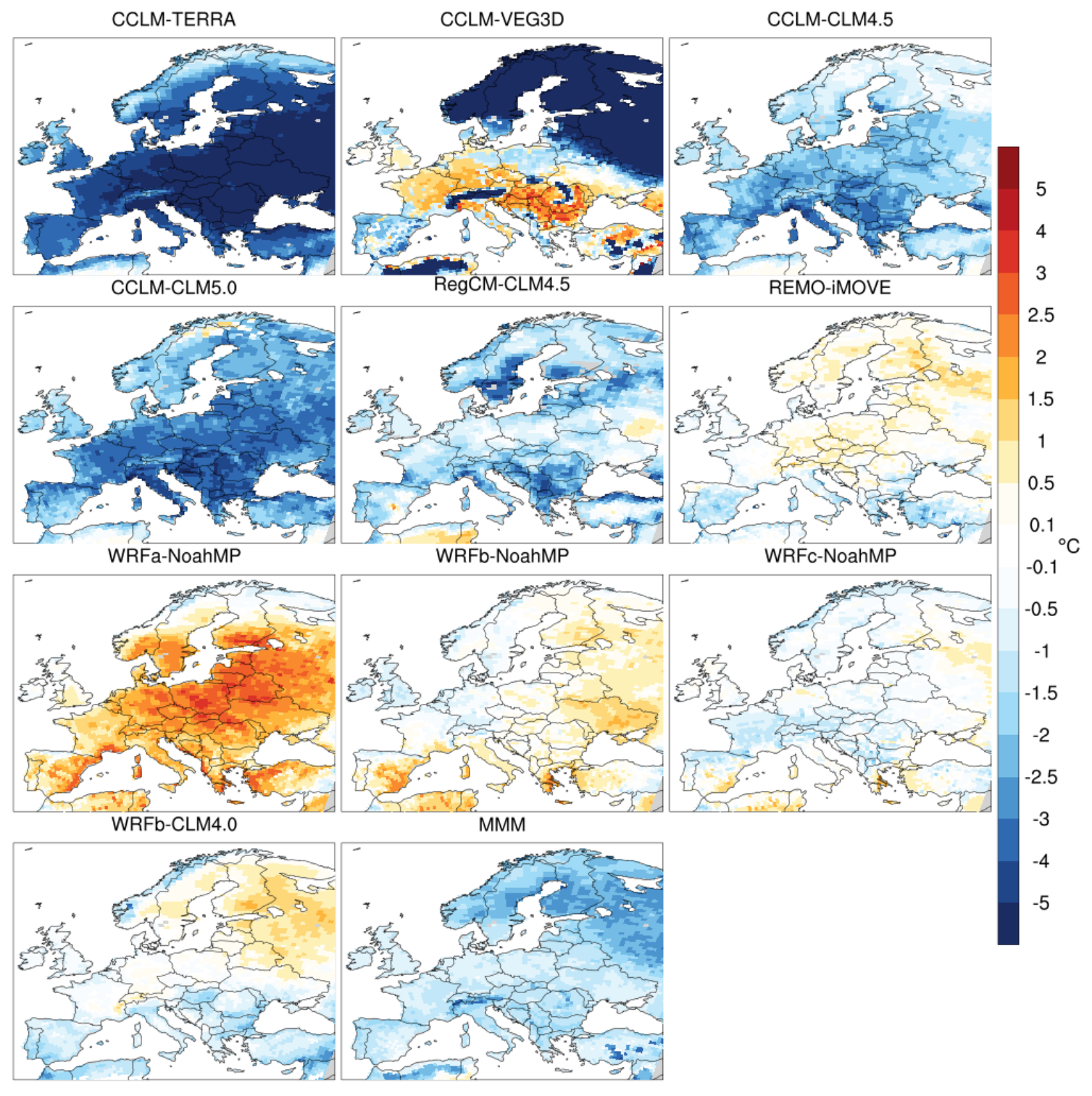

Figure S2: Afforestation (FOREST minus GRASS) impact on the annual amplitude of soil temperature (AAST) at $2 \mathrm{~cm}$ soil depth. MMM: Multi-Model-Mean. 


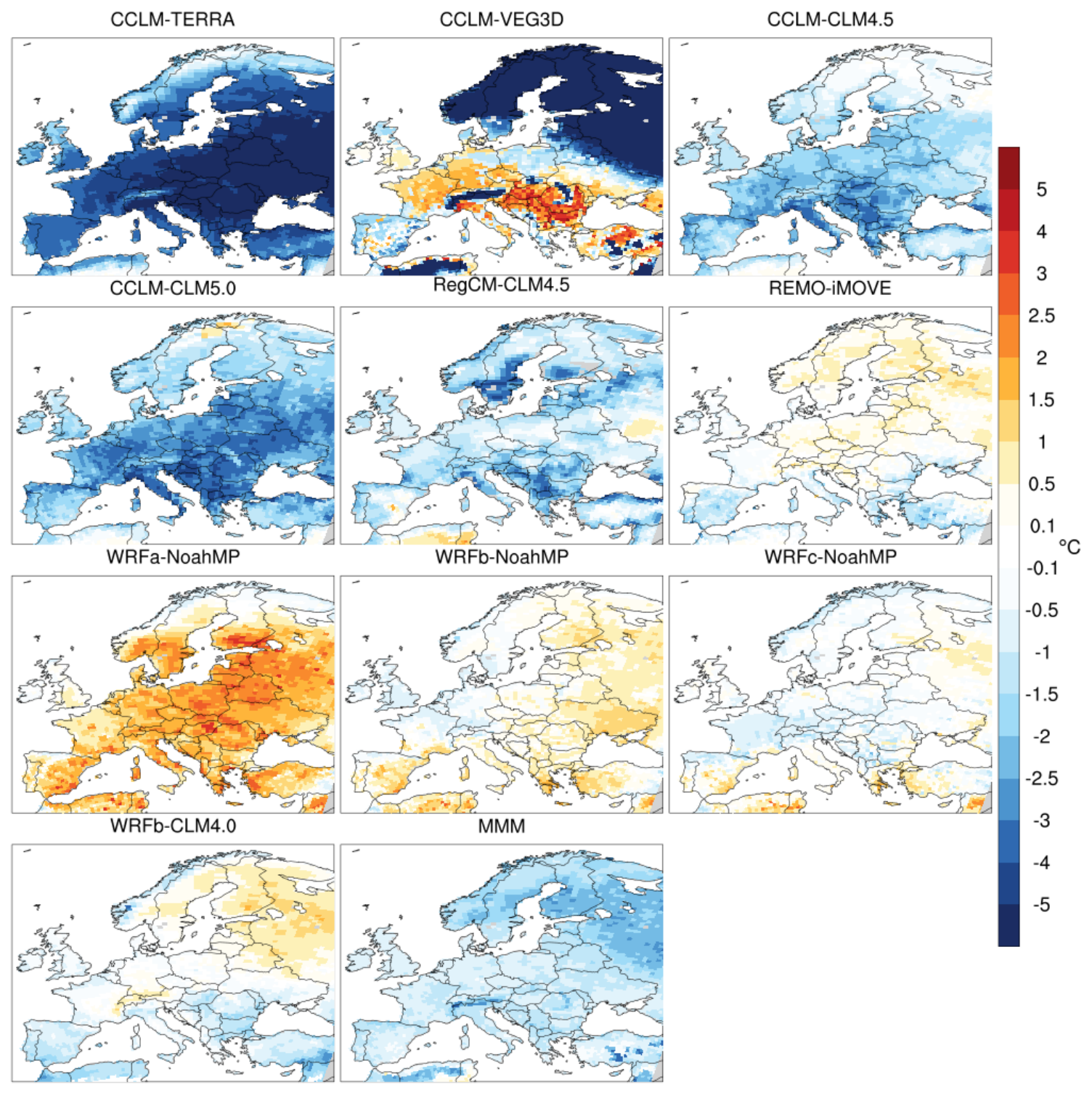

Figure S3: Afforestation (FOREST minus GRASS) impact on the annual amplitude of soil temperature (AAST) at $20 \mathrm{~cm}$ soil depth. MMM: Multi-Model-Mean. 


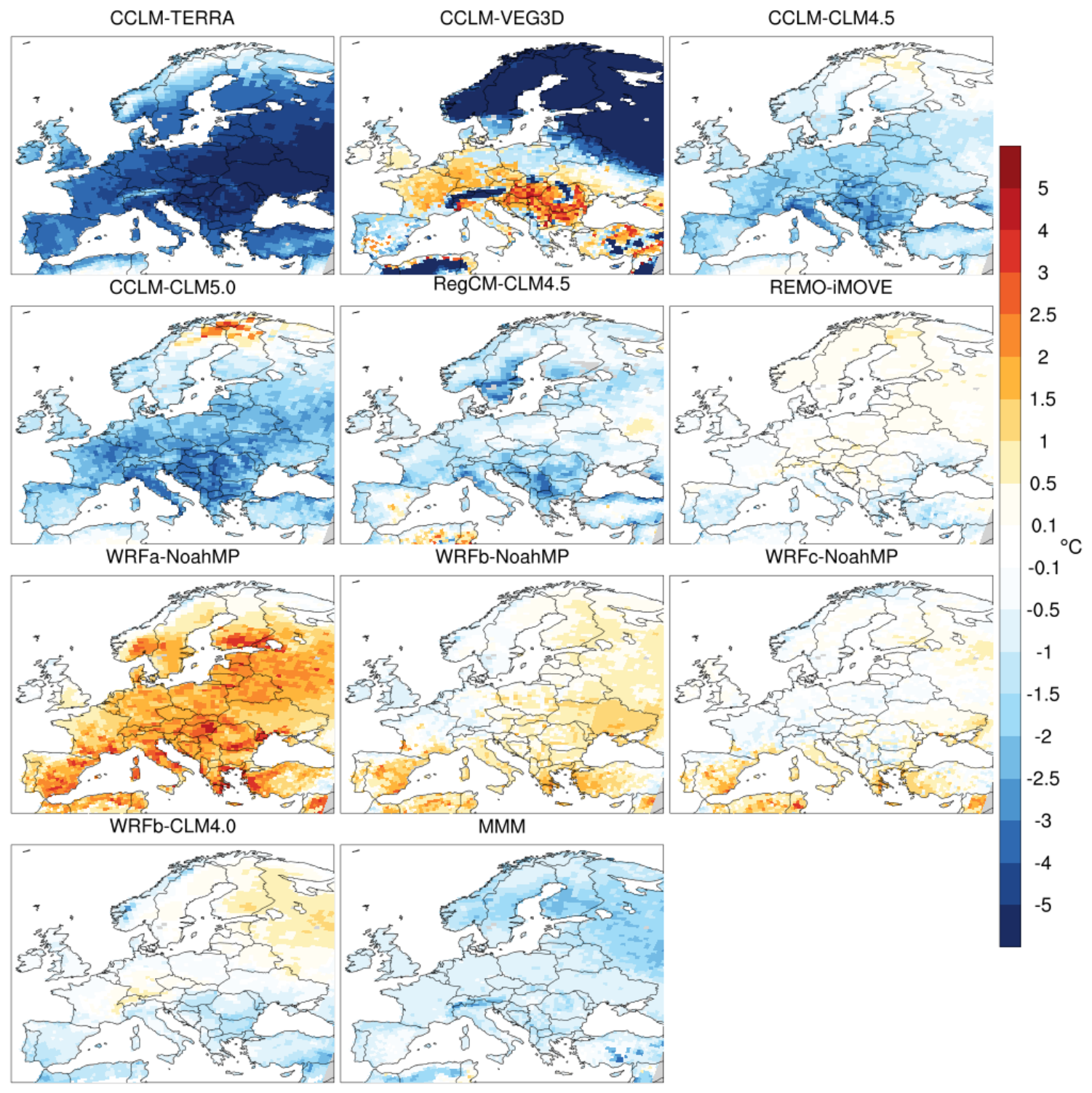

Figure S4: Afforestation (FOREST minus GRASS) impact on the annual amplitude of soil temperature (AAST) at $50 \mathrm{~cm}$ soil depth. MMM: Multi-Model-Mean. 
$\mathrm{AL}$
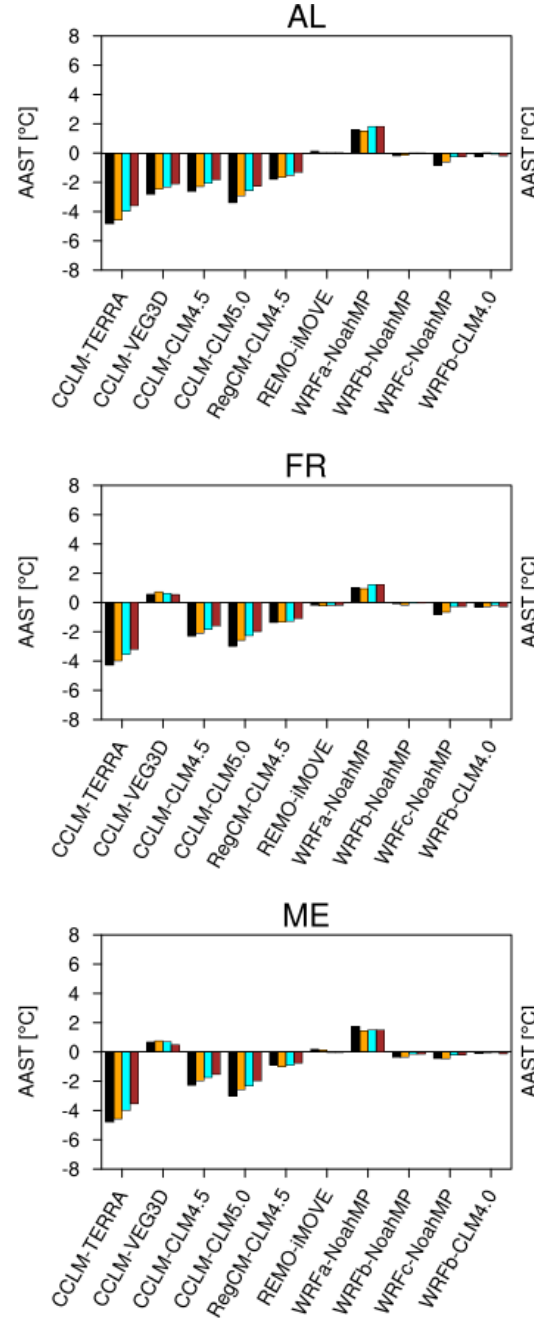

$\mathrm{BI}$

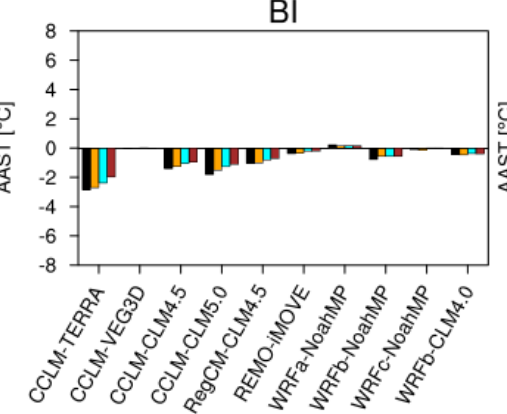

IP

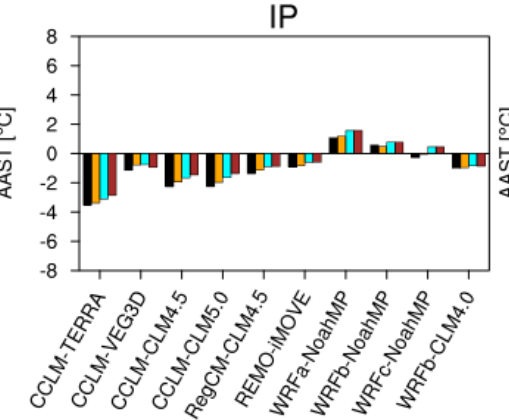

$\mathrm{SC}$

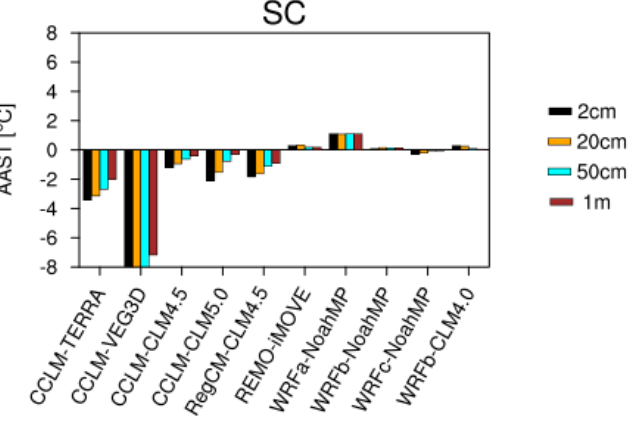

Figure S5: Afforestation (FOREST minus GRASS) impact on the annual amplitude of soil temperature (AAST) at $2 \mathrm{~cm}, 20 \mathrm{~cm}, 50 \mathrm{~cm}$ and 1 meter soil depth, summarized over all European regions. 

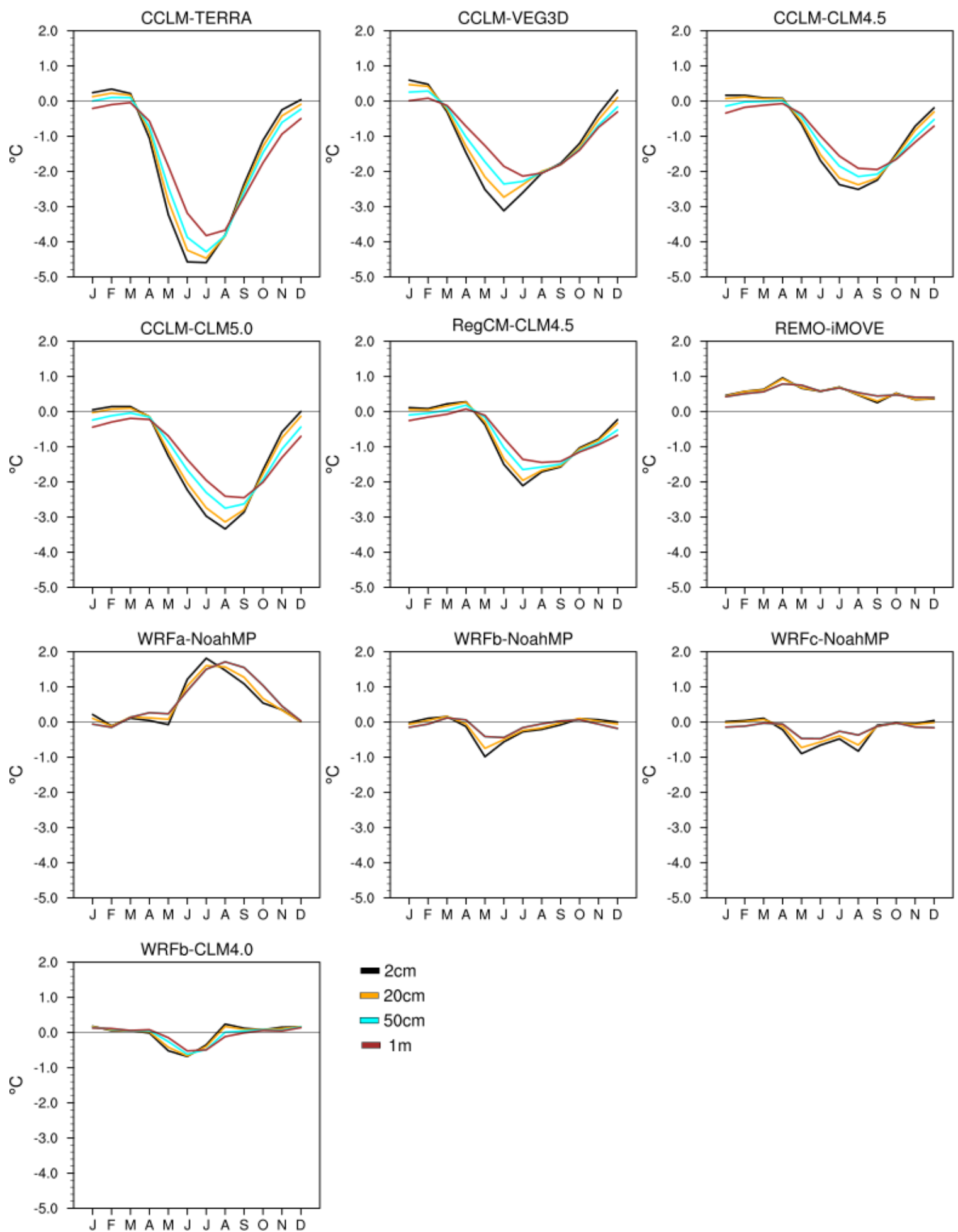

$$
\begin{aligned}
& -2 \mathrm{~cm} \\
& -20 \mathrm{~cm} \\
& =50 \mathrm{~cm} \\
& =1 \mathrm{~m}
\end{aligned}
$$

Figure S6: Afforestation impact (FOREST minus GRASS) on mean monthly soil temperature at four different soil depths over Alps. 

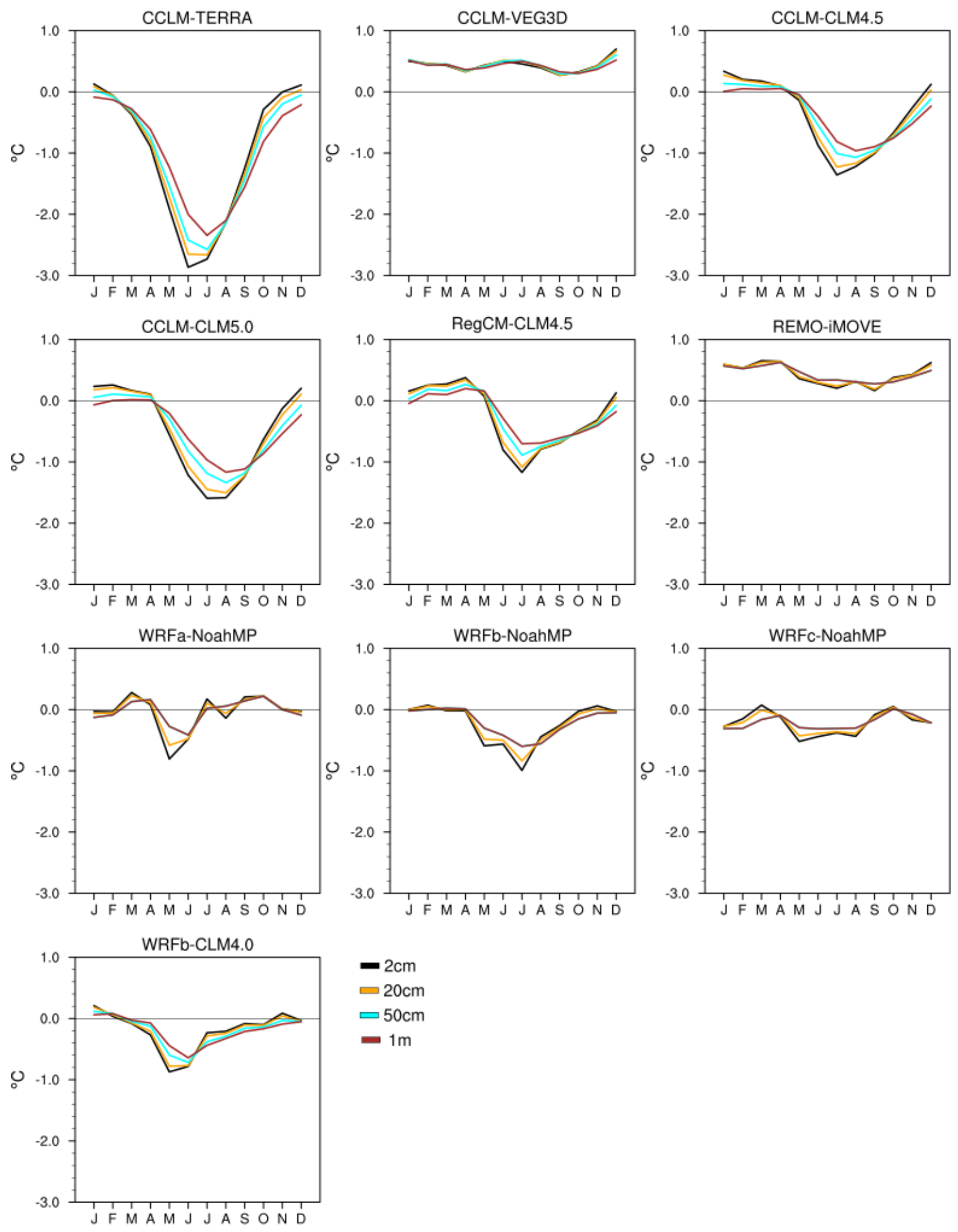

Figure S7: Afforestation impact (FOREST minus GRASS) on mean monthly soil temperature at four different soil depths over British Isles. 

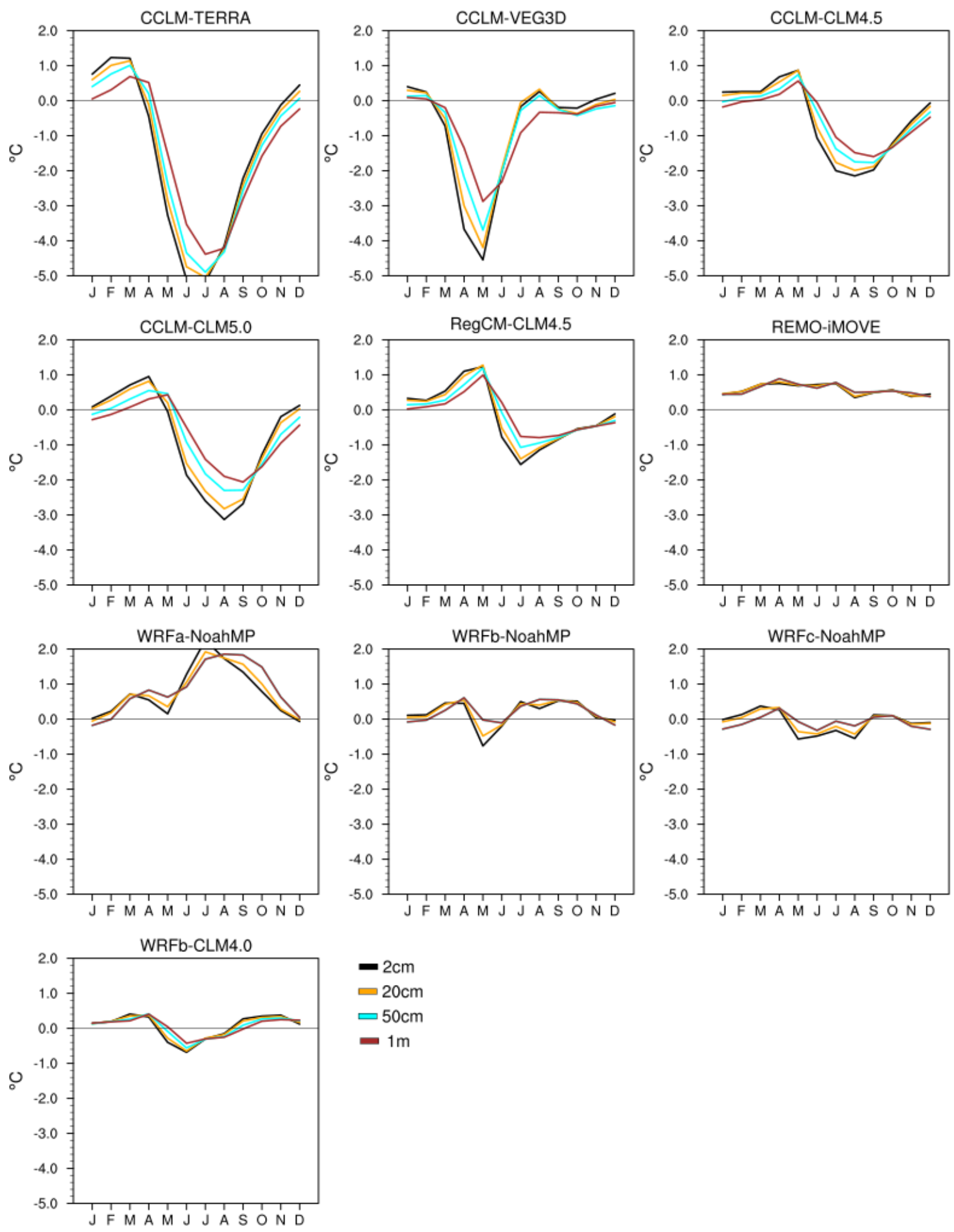

Figure S8: Afforestation impact (FOREST minus GRASS) on mean monthly soil temperature at four different soil depths over Eastern Europe. 

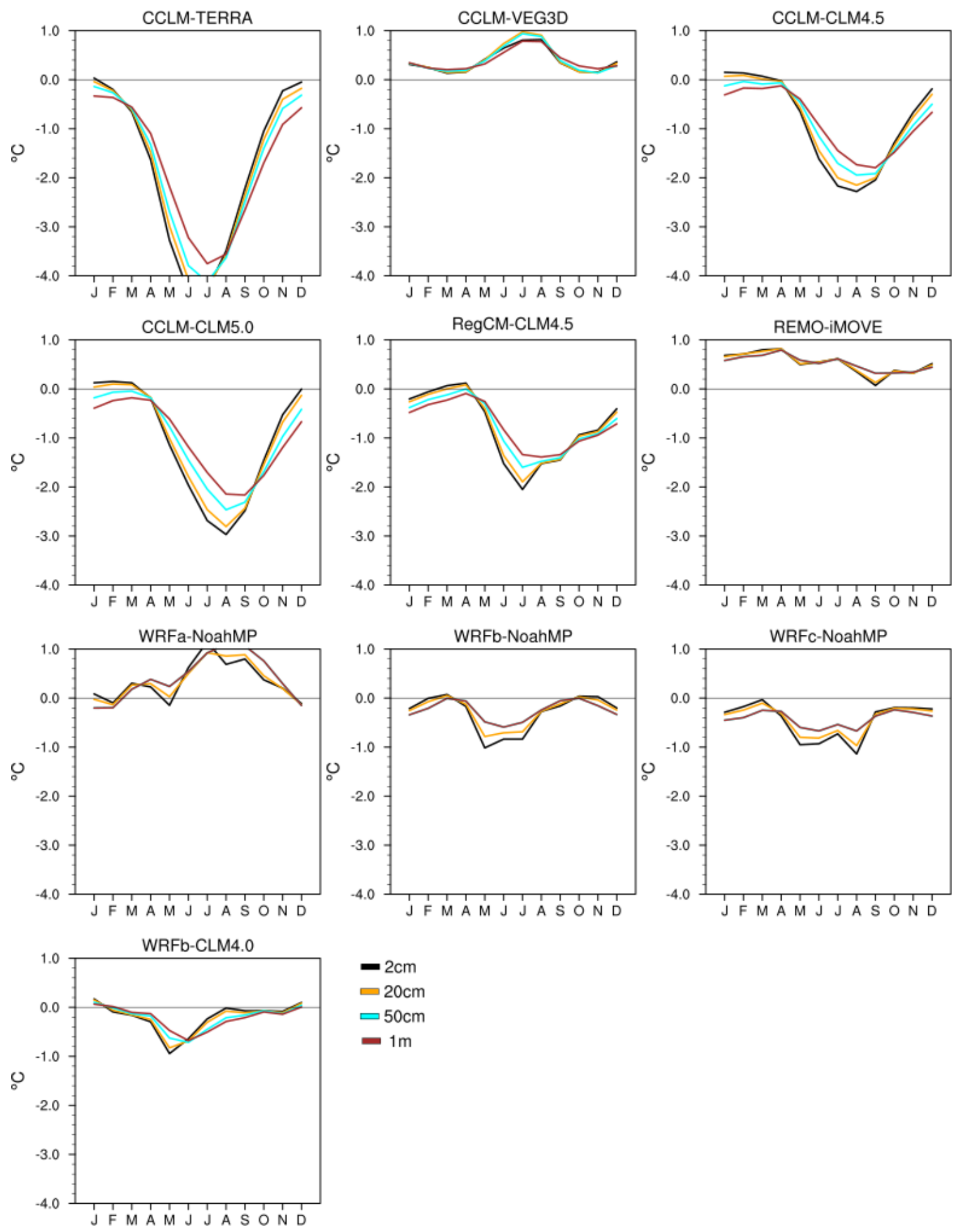

Figure S9: Afforestation impact (FOREST minus GRASS) on mean monthly soil temperature at four different soil depths over France. 

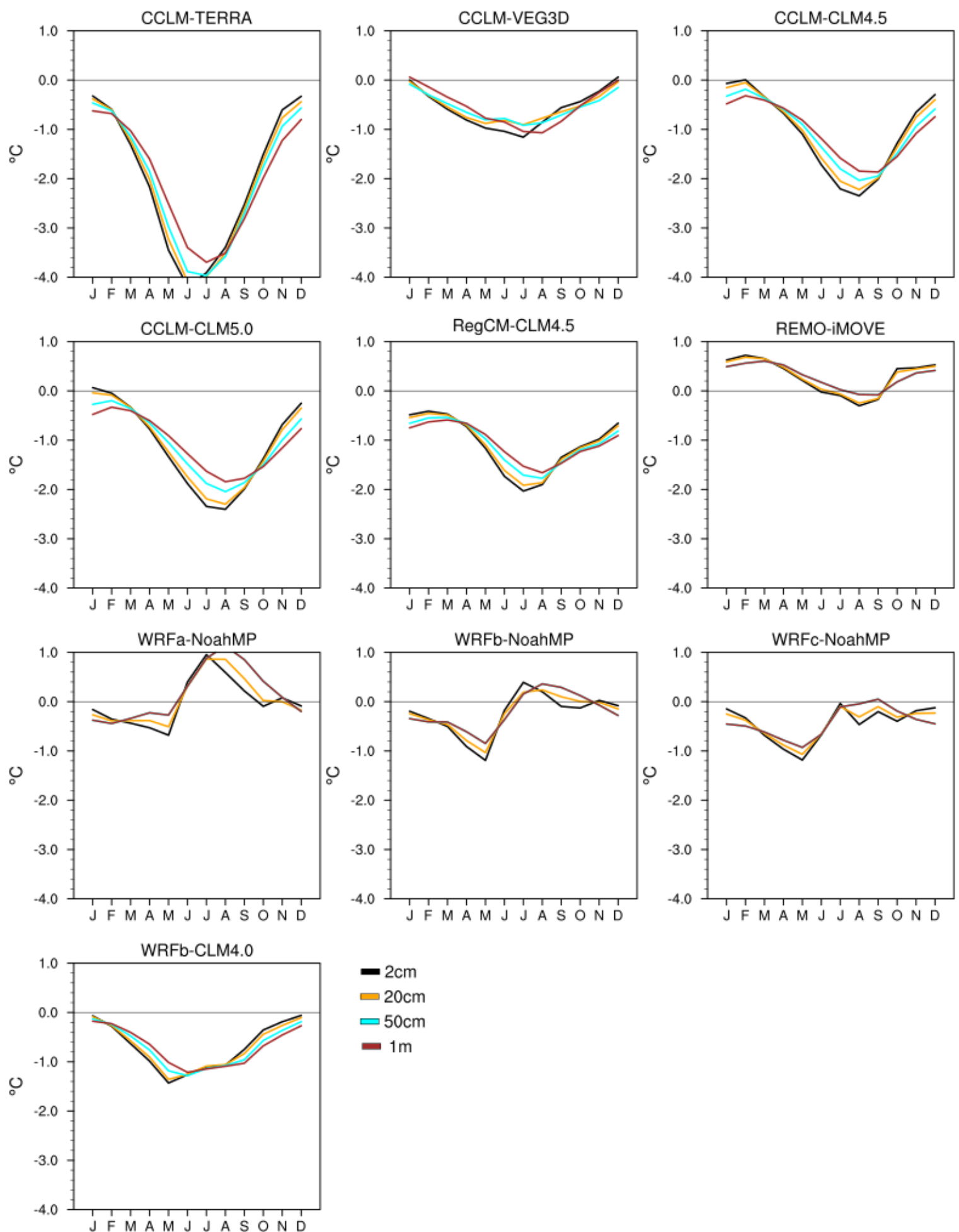

$$
\begin{aligned}
& =2 \mathrm{~cm} \\
& =20 \mathrm{~cm} \\
& =50 \mathrm{~cm} \\
& =1 \mathrm{~m}
\end{aligned}
$$

Figure S10: Afforestation impact (FOREST minus GRASS) on mean monthly soil temperature at four different soil depths over Iberian Peninsula. 

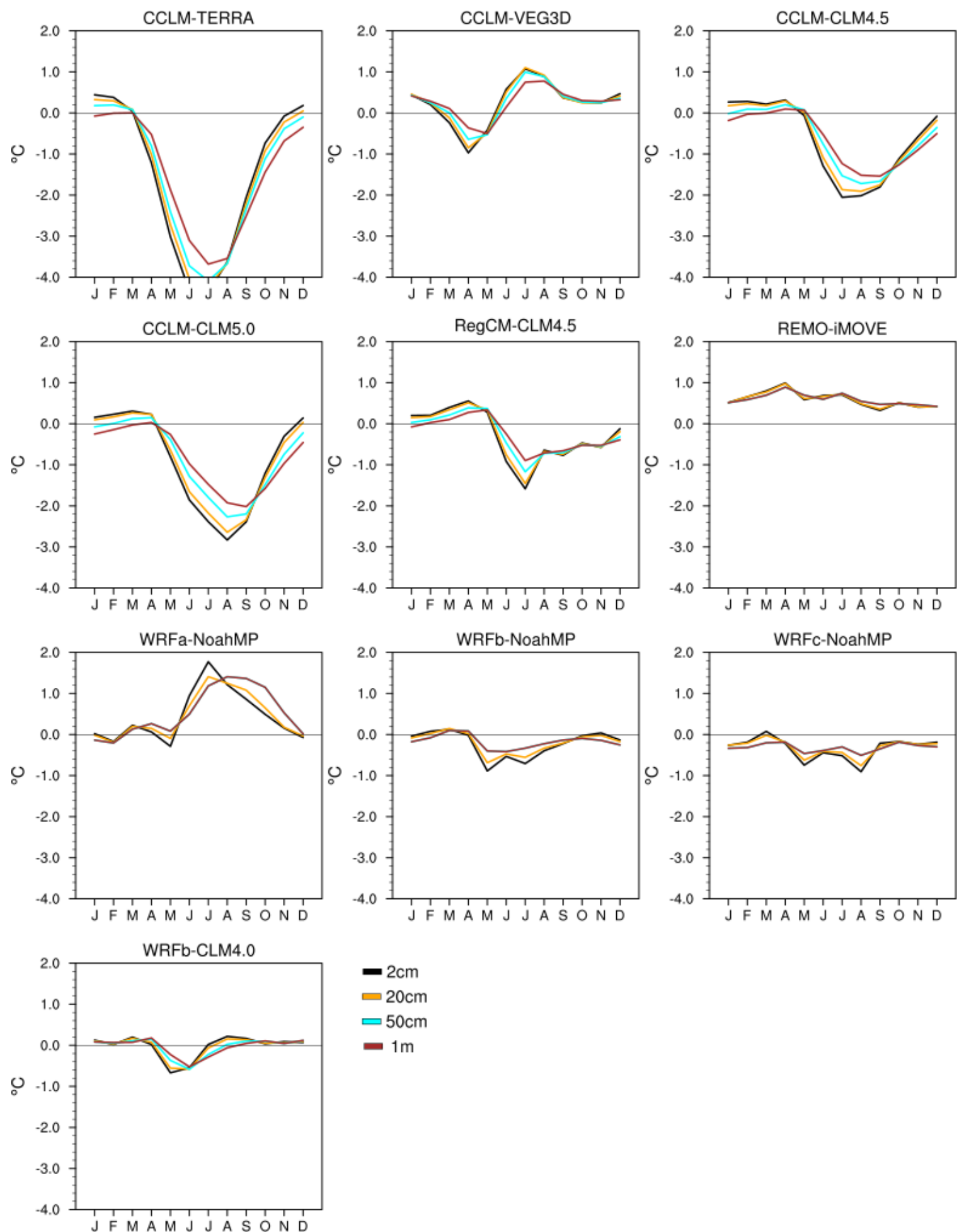

$$
\begin{aligned}
& =2 \mathrm{~cm} \\
& =20 \mathrm{~cm} \\
& =50 \mathrm{~cm} \\
& =1 \mathrm{~m}
\end{aligned}
$$

Figure S11: Afforestation impact (FOREST minus GRASS) on mean monthly soil temperature at four different soil depths over Mid-Europe. 

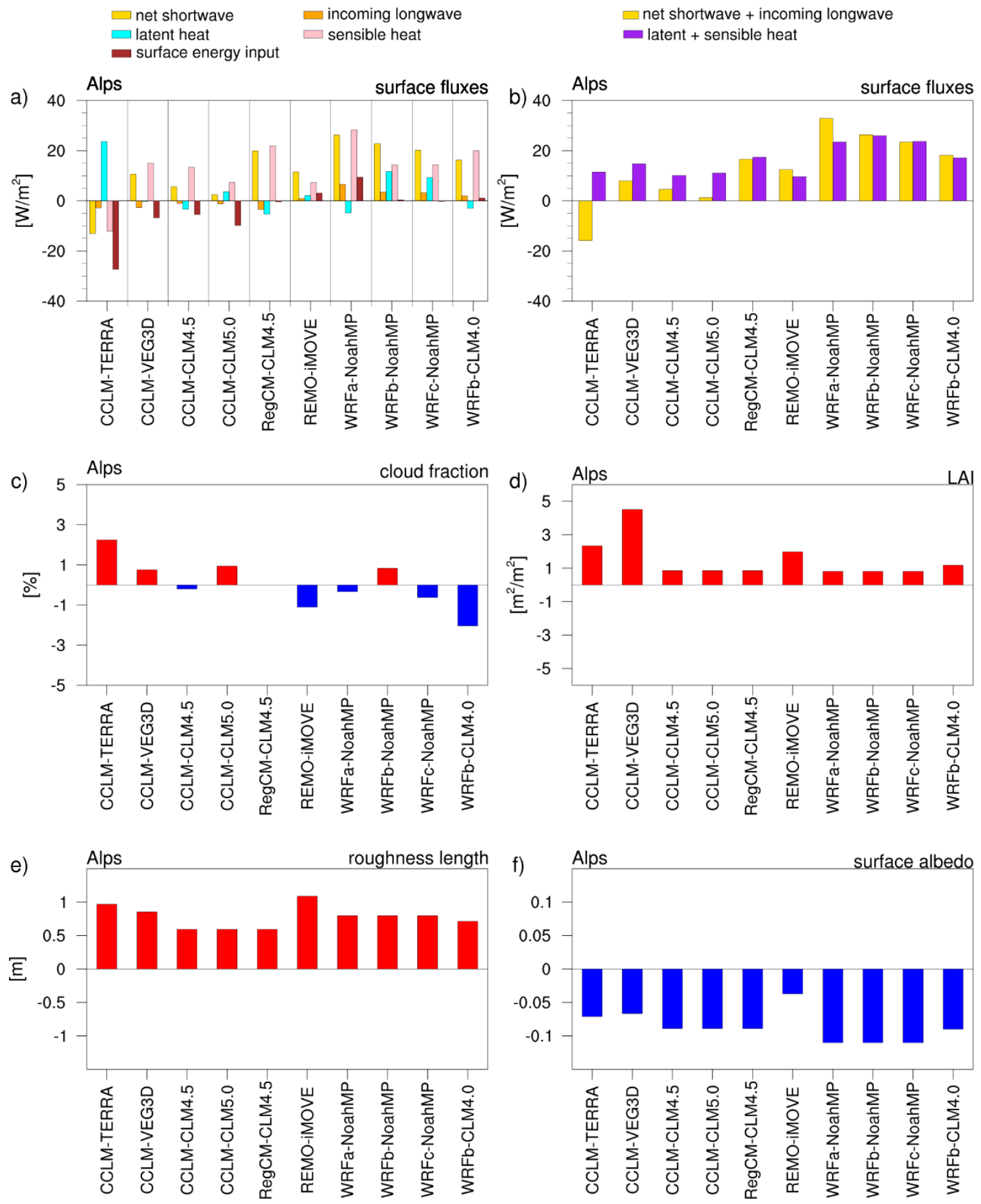

Figure S12: Changes in surface energy balance components (FOREST minus GRASS) averaged over Alps in summer, (b) The changes in available radiative energy at surface and in the sum of turbulent heat fluxes with afforestation (FOREST minus GRASS), (c) Cloud fraction response to afforestation across models, and the inter-model differences in leaf area index (LAI) (d), surface roughness (e) and surface albedo (f) in summer (yearly maximum). Positive (negative) values mean increase (decrease) with afforestation. 

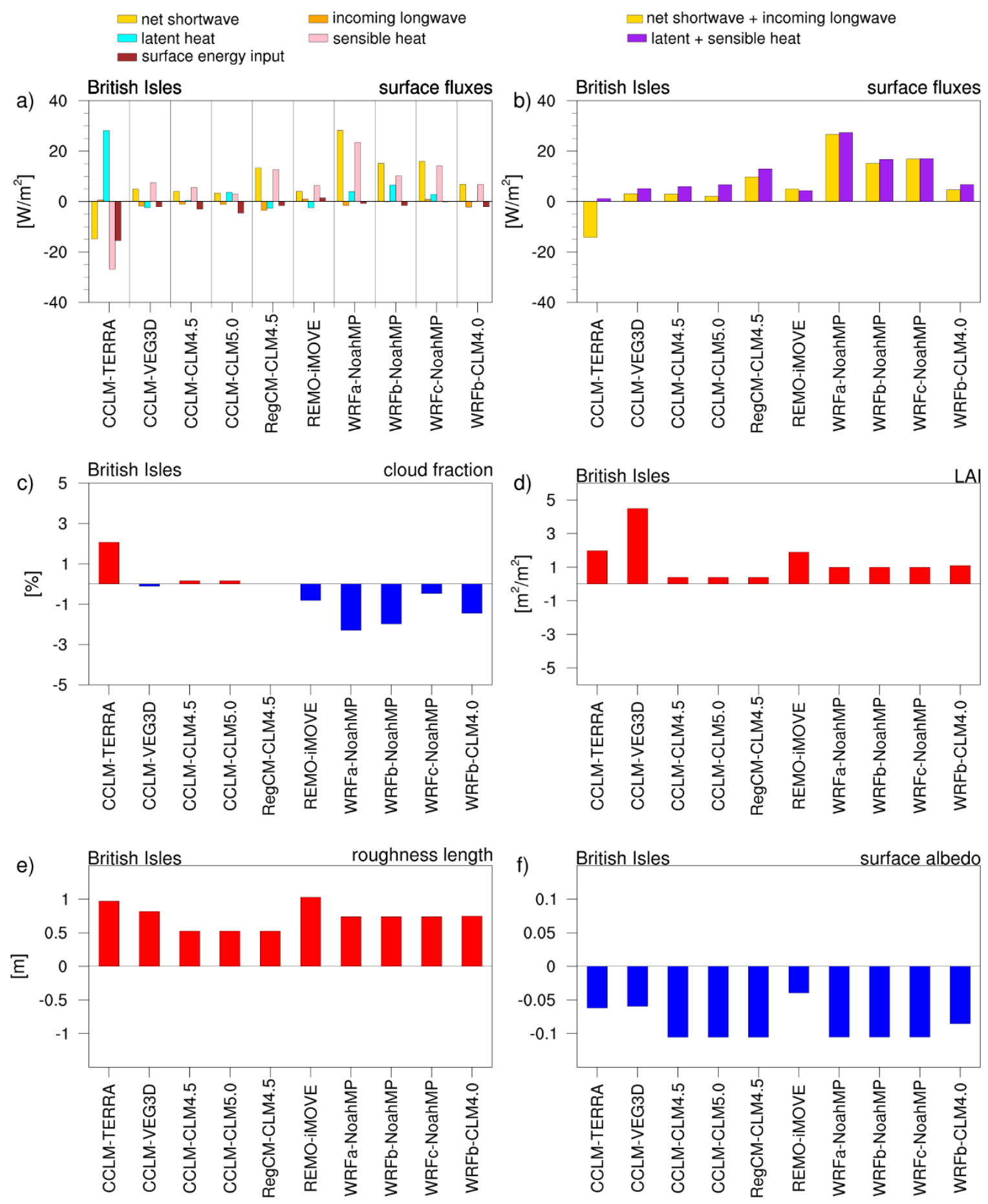

Figure S13: Changes in surface energy balance components (FOREST minus GRASS) averaged over British Isles in summer, (b) The changes in available radiative energy at surface and in the sum of turbulent heat fluxes with afforestation (FOREST minus GRASS), (c) Cloud fraction response to afforestation across models, and the inter-model differences in leaf area index (LAI) (d), surface roughness (e) and surface albedo (f) in summer (yearly maximum). Positive (negative) values mean increase (decrease) with afforestation. 
$\square$ net shortwave $\quad \square$ incoming longwave
latent heat
surface energy input

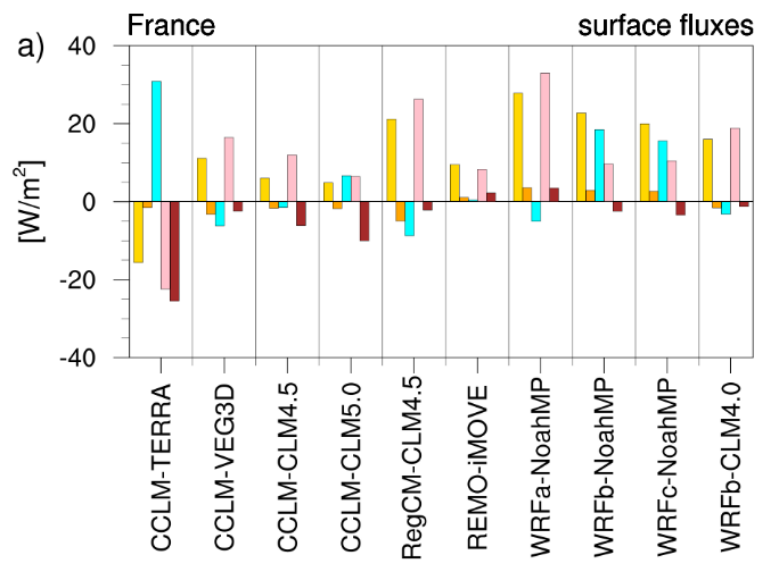

$\square$ net shortwave + incoming longwave

latent + sensible heat
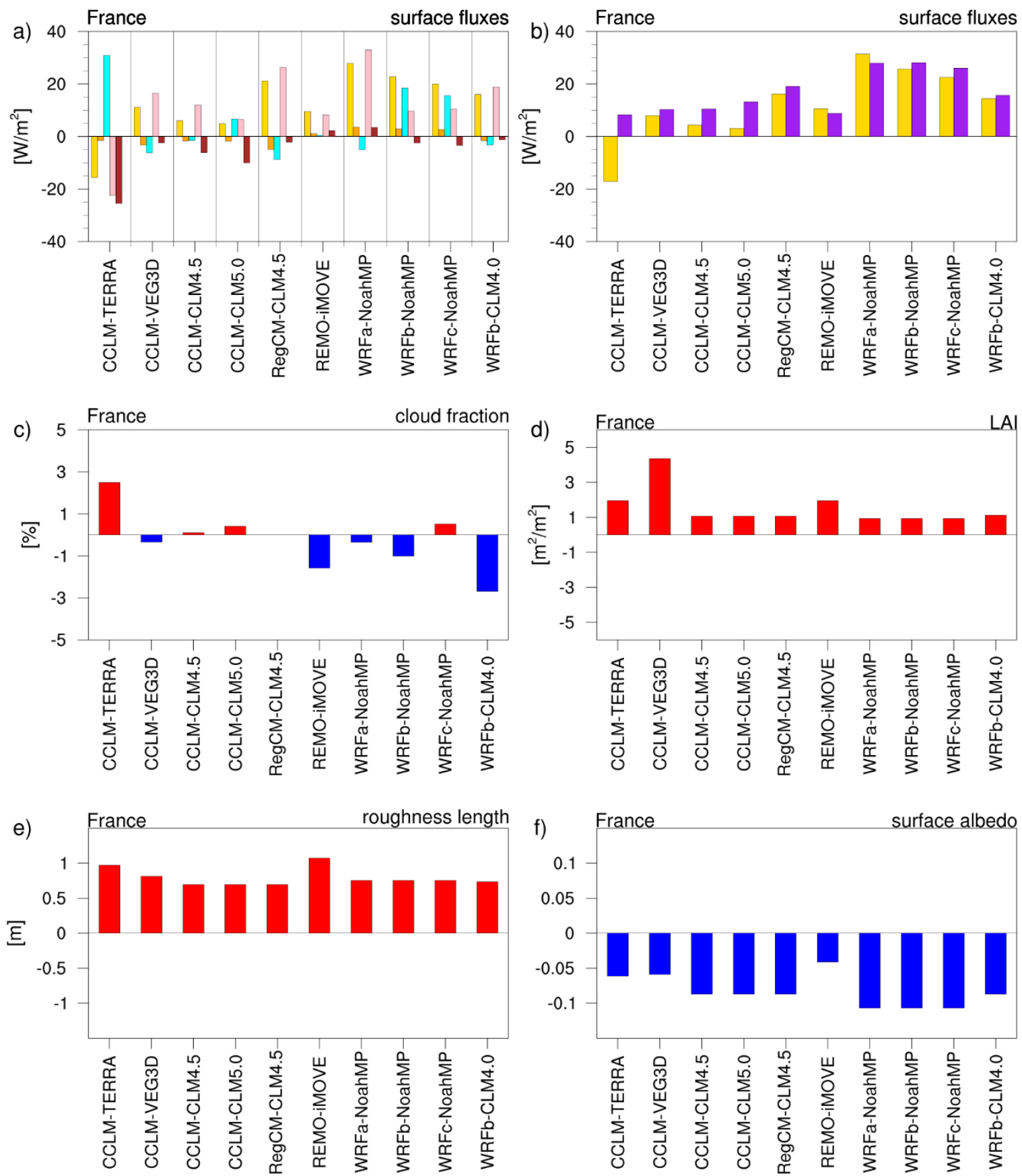

Figure S14: Changes in surface energy balance components (FOREST minus GRASS) averaged over France in summer, (b) The changes in available radiative energy at surface and in the sum of turbulent heat fluxes with afforestation (FOREST minus GRASS), (c) Cloud fraction response to afforestation across models, and the inter-model differences in leaf area index (LAI) (d), surface roughness (e) and surface albedo (f) in summer (yearly maximum). Positive (negative) values mean increase (decrease) with afforestation. 

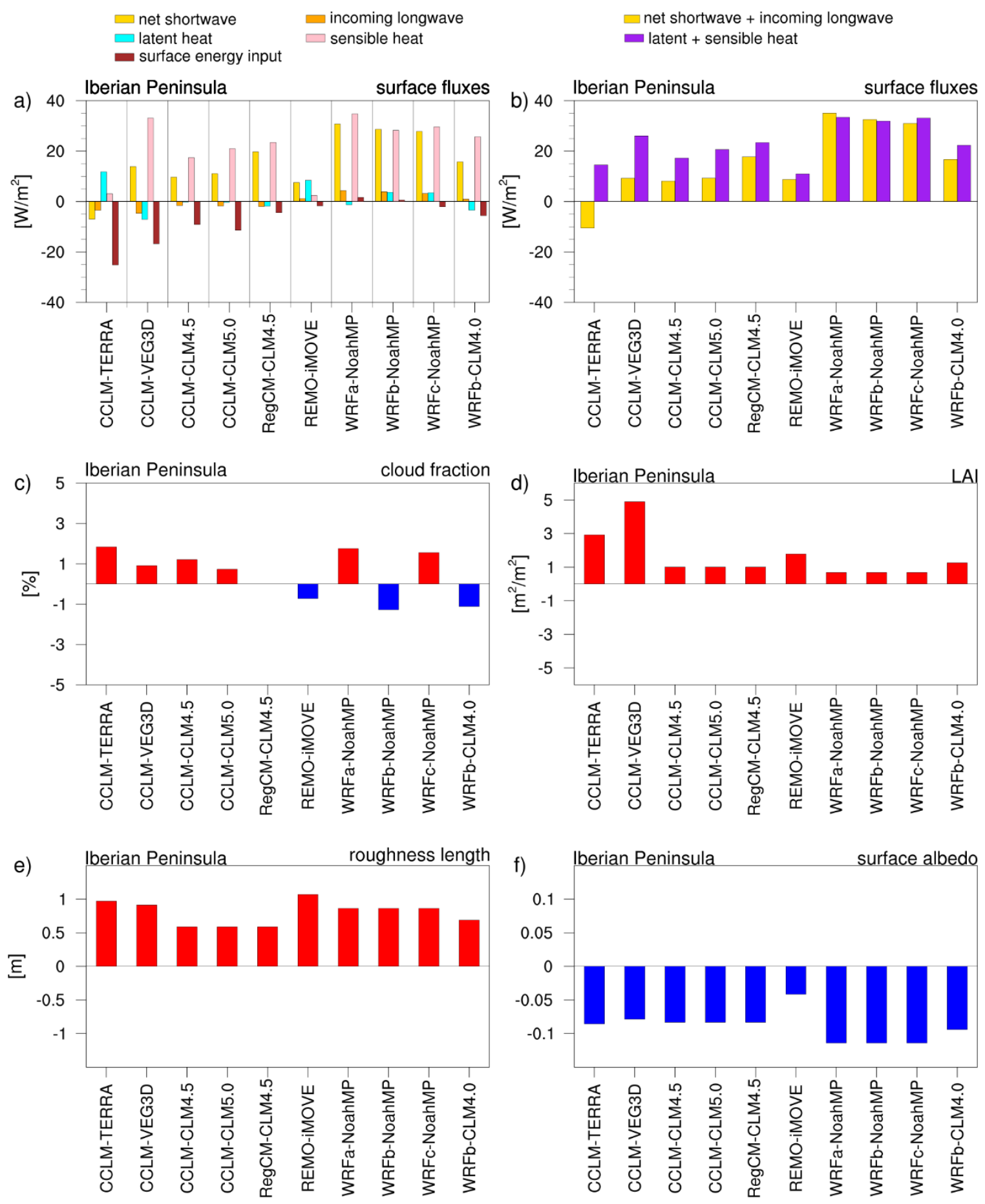

Figure S15: Changes in surface energy balance components (FOREST minus GRASS) averaged over Iberian Peninsula in summer, (b) The changes in available radiative energy at surface and in the sum of turbulent heat fluxes with afforestation (FOREST minus GRASS), (c) Cloud fraction response to afforestation across models, and the inter-model differences in leaf area index (LAI) (d), surface roughness (e) and surface albedo (f) in summer (yearly maximum). Positive (negative) values mean increase (decrease) with afforestation. 


$$
\begin{aligned}
& \square \text { net shortwave } \square \text { incoming longwave } \\
& \text { latent heat } \\
& \text { surface energy input }
\end{aligned}
$$
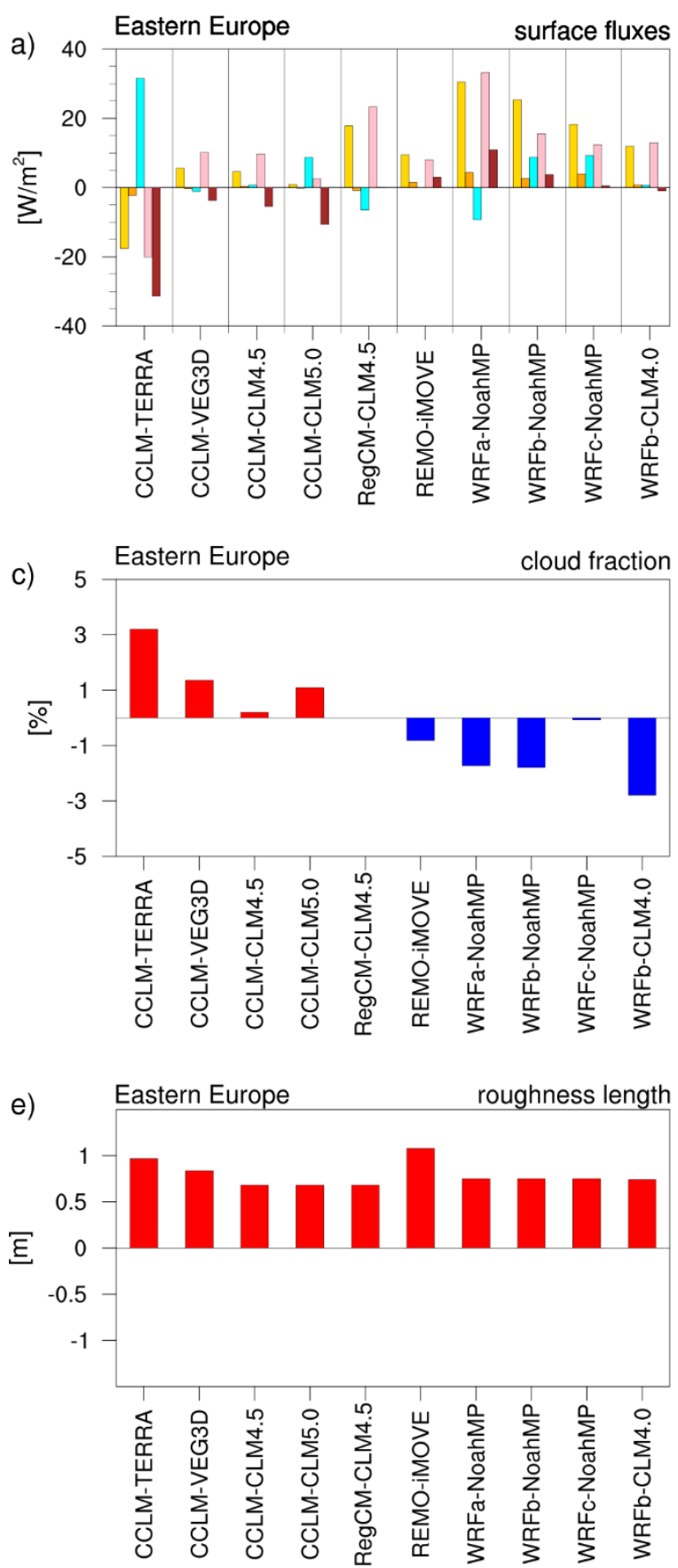
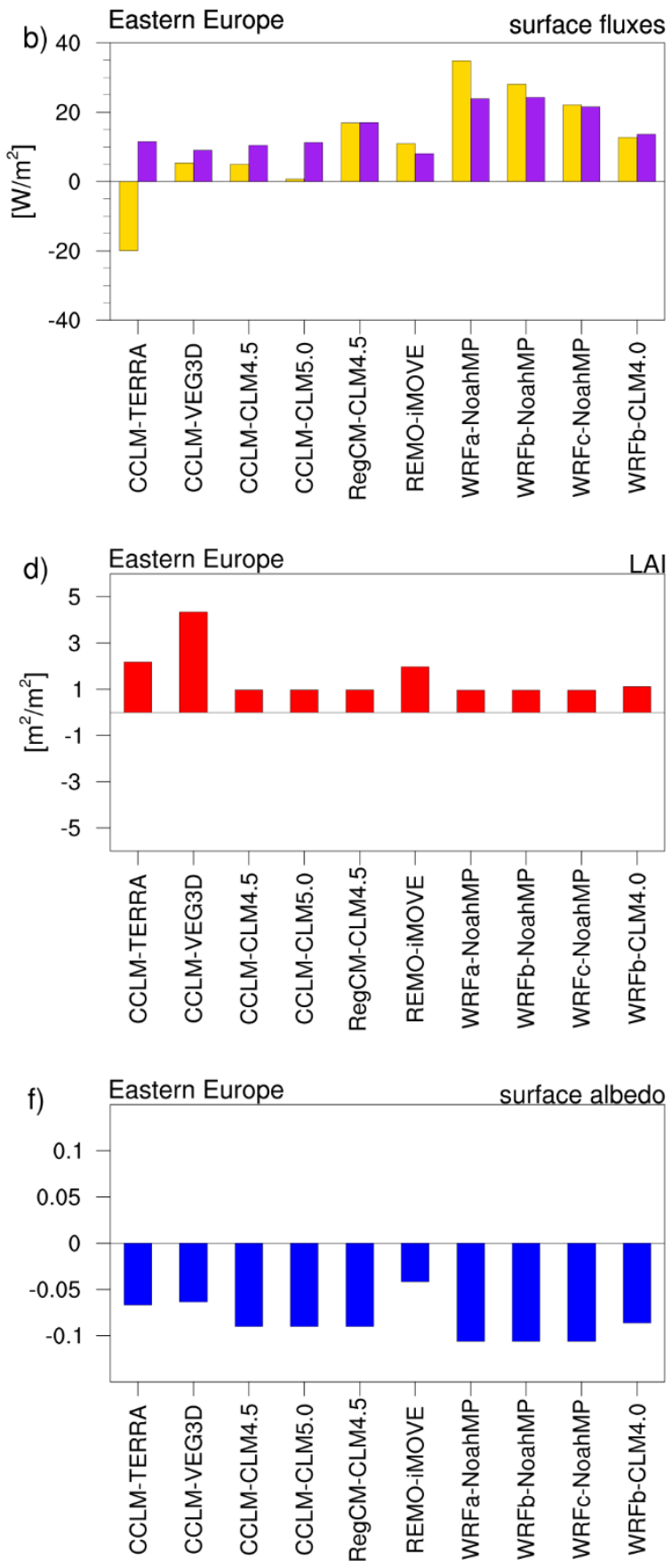

Figure S16: Changes in surface energy balance components (FOREST minus GRASS) averaged over Eastern Europe in summer, (b) The changes in available radiative energy at surface and in the sum of turbulent heat fluxes with afforestation (FOREST minus GRASS), (c) Cloud fraction response to afforestation across models, and the inter-model differences in leaf area index (LAI) (d), surface roughness (e) and surface albedo (f) in summer (yearly maximum). Positive (negative) values mean increase (decrease) with afforestation. 


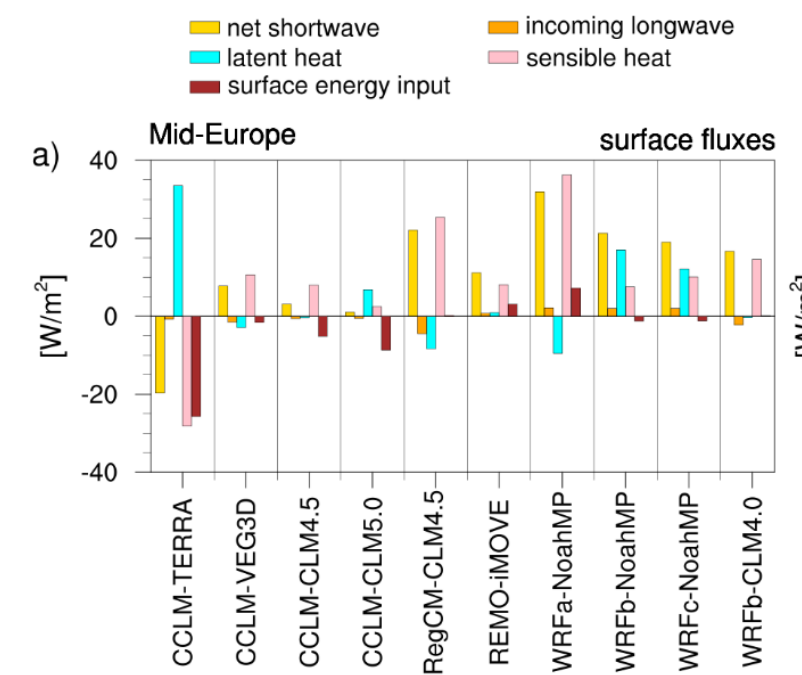
$\square$ net shortwave + incoming longwave
latent + sensible heat
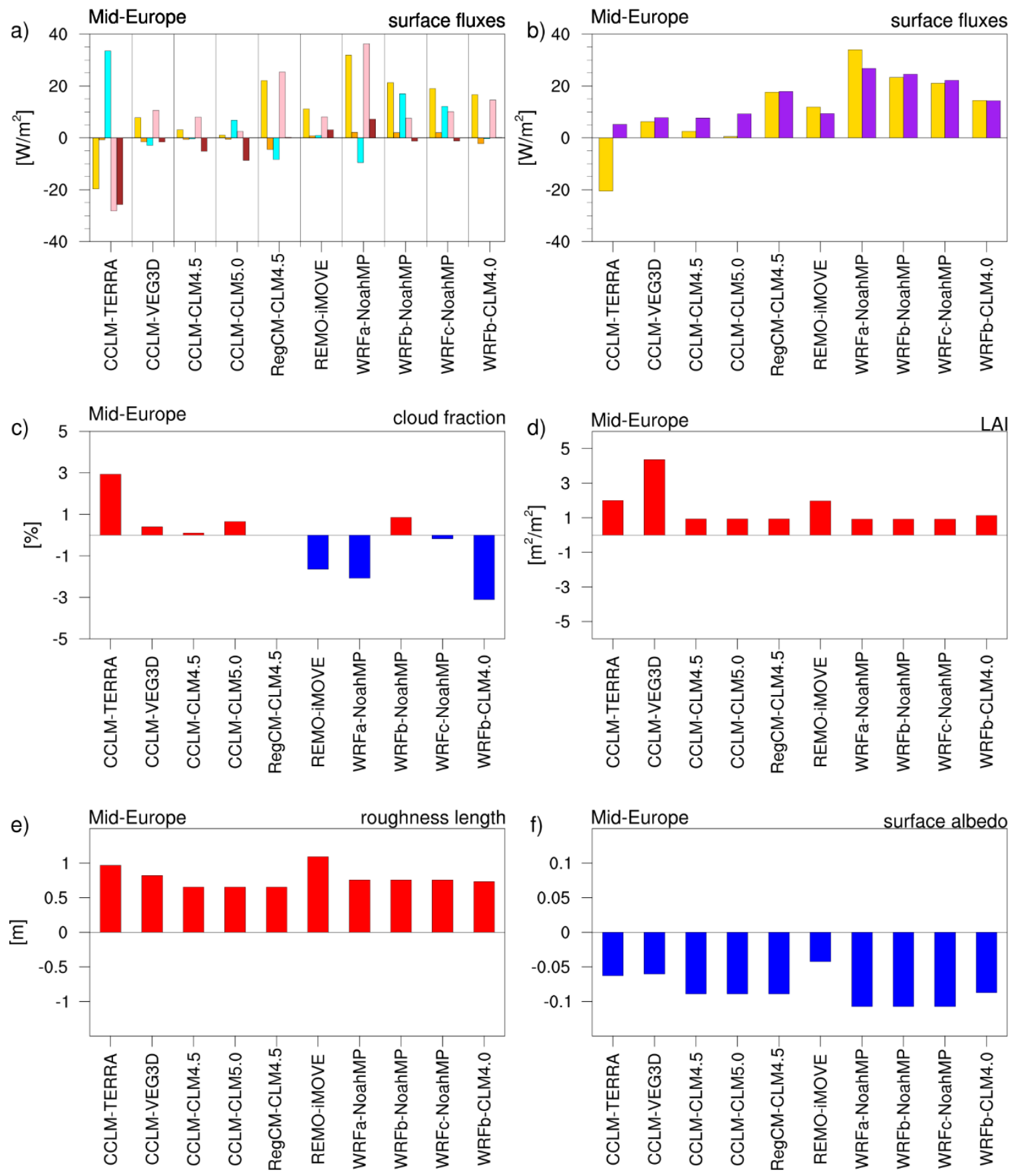

Figure S17: Changes in surface energy balance components (FOREST minus GRASS) averaged over Mid-Europe in summer, (b) The changes in available radiative energy at surface and in the sum of turbulent heat fluxes with afforestation (FOREST minus GRASS), (c) Cloud fraction response to afforestation across models, and the inter-model differences in leaf area index (LAI) (d), surface roughness (e) and surface albedo (f) in summer (yearly maximum). Positive (negative) values mean increase (decrease) with afforestation. 

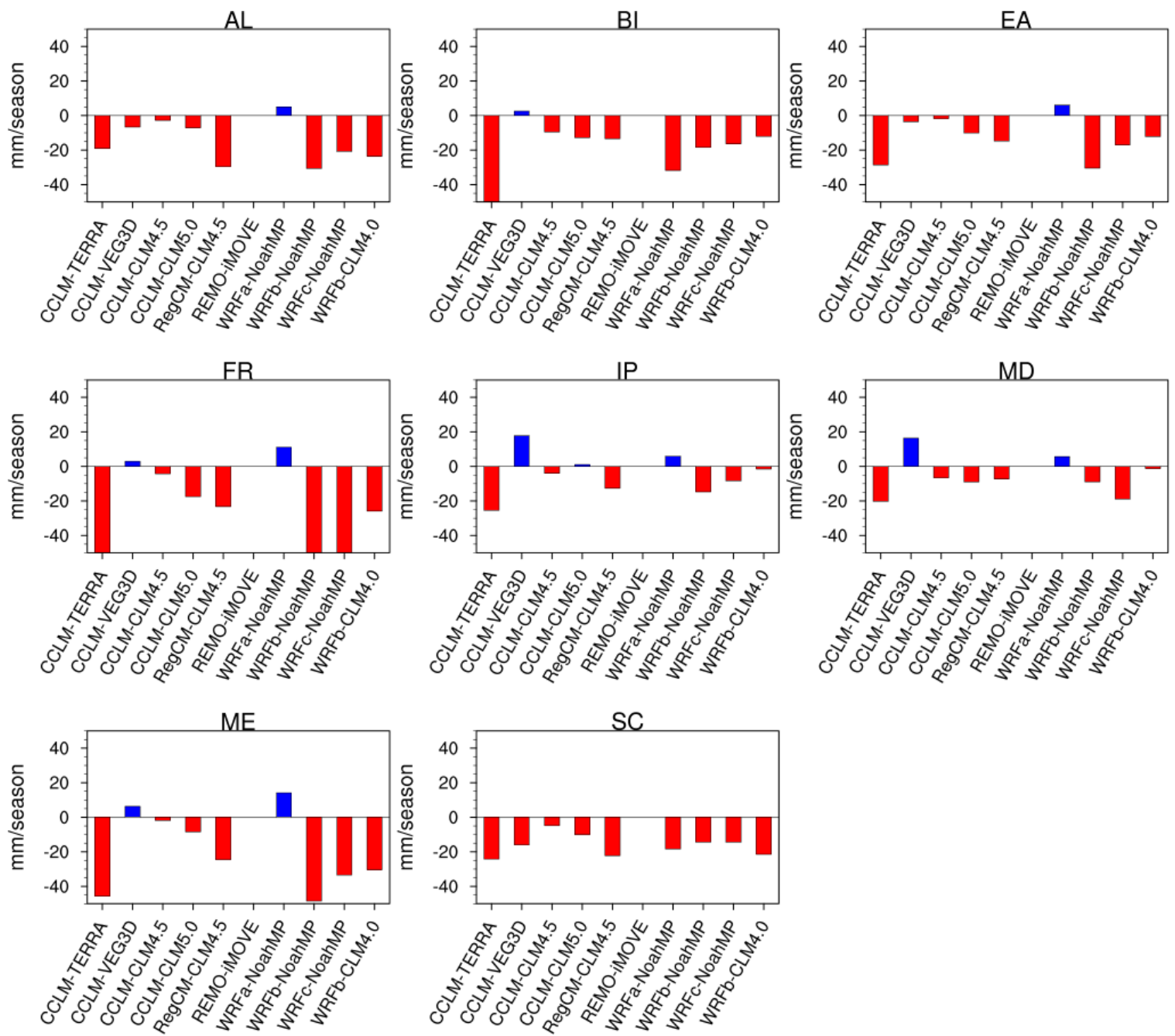

Figure S18: Afforestation (FOREST minus GRASS) impact on surface water balance, defined as the difference between precipitation and evapotranspiration, during summer season over the regions of interest. 

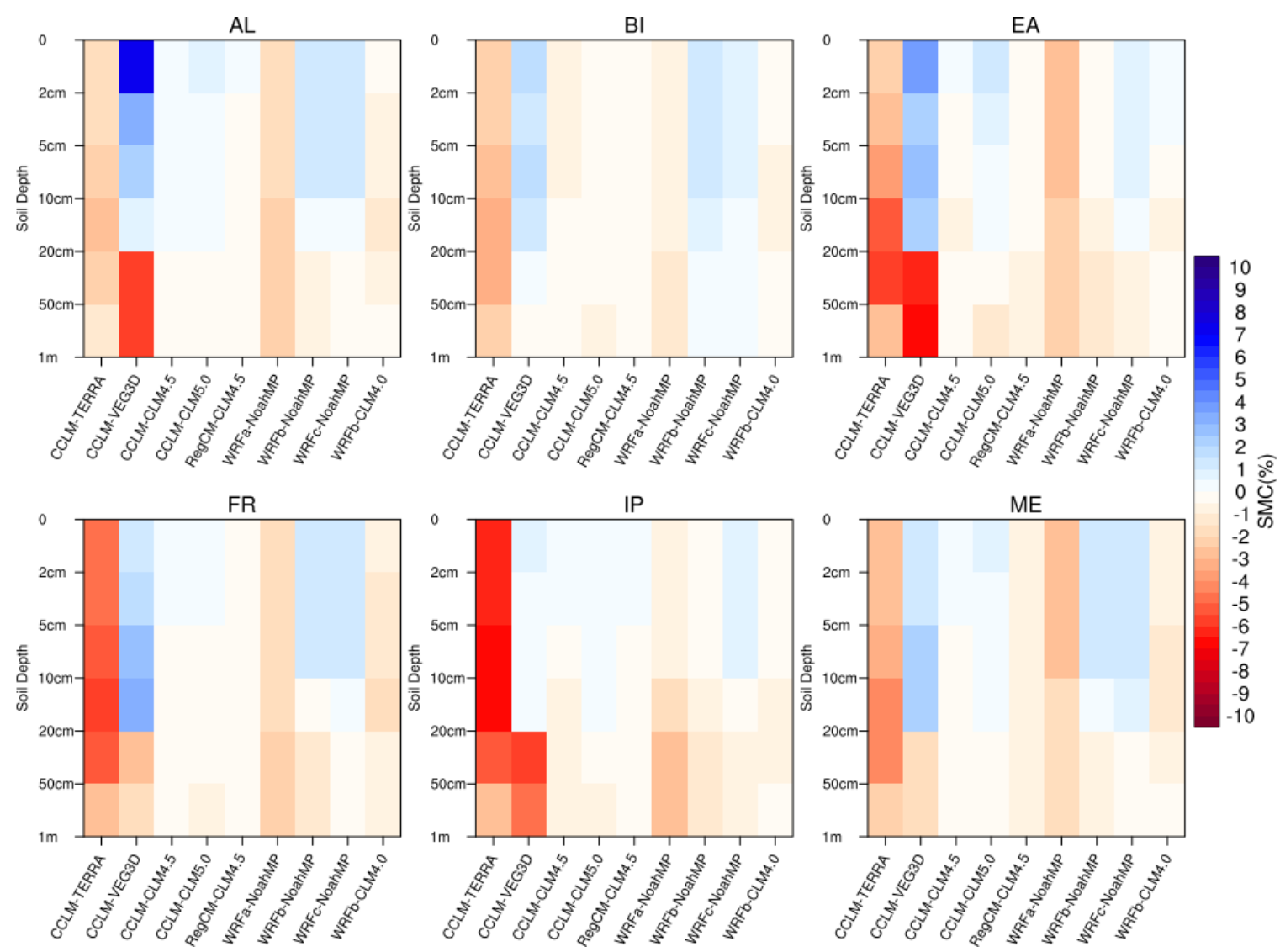

Figure S19: Summer changes in soil moisture content (SMC) due to afforestation (FOREST minus GRASS) in the top 1 meter of the soil over European sub-regions. 